\title{
EXISTENCE AND STABILITY OF A SCREW DISLOCATION UNDER ANTI-PLANE DEFORMATION
}

\author{
T. HUDSON AND C. ORTNER
}

\begin{abstract}
We formulate a variational model for a geometrically necessary screw dislocation in an anti-plane lattice model at zero temperature. Invariance of the energy functional under lattice symmetries renders the problem non-coercive. Nevertheless, by establishing coercivity with respect to the elastic strain and a concentration compactness principle, we prove existence of a global energy minimiser and thus demonstrate that dislocations are globally stable equilibria within our model.
\end{abstract}

\section{INTRODUCTION}

Dislocations are line defects in crystalline solids which can be described by discontinuous displacements of a homogeneous crystal: to obtain the simplest forms of dislocations, the lattice is sliced along some half plane (the Volterra cut), and then deformed so that the lattice remains almost perfect away from the edge of that half plane (the dislocation line) [15, 36]. Since dislocations are the principal carriers of plastic flow in crystals [23, 27, 34], they are among the most widely studied objects of materials science. They have been investigated analytically as points in an elastic continuum [15, 7, 30, 13, 3, 6], as crystal defects using molecular simulation techniques [6, 32, 31], or through a variety of intermediate models such as phase field descriptions [19, 29, 14. We refer to [11, 15, 18, 6] for introductions to these various models.

In the present work, we focus on the atomistic structure of dislocations. Precisely, we shall demonstrate that they can be understood as global minima in a variational problem. To the best of our knowledge, our results are the first that establish the existence of dislocations as stable equilibrium configurations of an atomistic energy.

Our work is motivated by ongoing efforts to develop multi-scale models of dislocations such as dislocation dynamics [6], and far-field coarse-graining techniques such as quasicontinuum and related methods [22, 31, 33]. Our results contribute to a precise qualitative understanding of the atomistic structure of dislocations, which can be used to inform the formulation and analysis of such multi-scale schemes [25, 20].

Further, our work is inspired by a recent effort to place the theory of dislocations on a rigorous mathematical foundation, in particular clarifying the connections between the various models mentioned above [21, 37, 28, 12. We outline only a small fraction of the contributions here, most closely related to our own work. We believe that, to some extent, our results help to overcome simplifying assumptions made in many of these works.

Possibly the most complete analysis of a static model of dislocations is provided in [12, 14, 8]. This series of papers studies a continuum phase field model for dislocations in a periodic environment of pinning sites first described in [19]. The authors obtain a variety of scaling regimes, depending upon the number of the pinning sites relative to their size in terms of the interatomic spacing.

Date: April 21, 2021.

2000 Mathematics Subject Classification. 74G25, 74G65, 70C20, 49J45, 74M25, 74E15.

Key words and phrases. Screw dislocations, anti-plane shear, lattice models, concentration compactness.

CO was supported by the EPSRC grant EP/H003096 "Analysis of atomistic-to-continuum coupling methods". TH was supported by the UK EPSRC Science and Innovation award to the Oxford Centre for Nonlinear PDE $(\mathrm{EP} / \mathrm{E} 035027 / 1)$ 
A mathematically consistent description of dislocations in an atomistic setting, using the language of algebraic topology is given in [4]. The concepts and language developed therein are used to derive models of the elastic energy of a dislocation configuration. A rigorous discrete-to-continuum passage within this framework is established in [28] using the language of $\Gamma$-convergence. Related works analysing dislocations and other similar defects in discrete systems are [1, 2].

The works cited above concerning discrete and semidiscrete models of screw dislocations are primarily concerned with asymptotics of the elastic stored energy, given a number of prescribed dislocation cores. The creation or destruction of additional cores, for example via the introduction of dipoles, is either forbidden or explicitly tracked in the energy functional through a term accounting for a positive core energy.

In contrast, our model allows for the creation and destruction of dislocation dipoles without any such penalty, accounting only for the stored elastic energy. More precisely, we consider a static atomistic model for screw dislocations in a similar vein to [28], and show that unconstrained stable equilibrium configurations containing dislocations exist. Although we do not pursue this in the present work, we observe that the analytic properties of the equilibria we obtain should allow for a natural extension of the results in [28] to our unconstrained model.

1.1. Outline. We consider anti-plane displacements of a BCC crystal, in the direction of a screw dislocation line (that is, parallel to the Burgers vector). For two displacements, $y, \tilde{y}$ we consider the energy difference

$$
E(y ; \tilde{y}):=\sum_{b \in \mathcal{B}}\left[\psi\left(D \tilde{y}_{b}\right)-\psi\left(D y_{b}\right)\right]
$$

where $\mathcal{B}$ is a set of pairs of interacting (lines of) atoms, $D y_{b}$ denotes a finite difference, and $\psi$ is a potential describing this interaction. The potential $\psi$ is 1 -periodic, where 1 is the atomic spacing, mimicking the behaviour of realistic pair interaction potentials. In particular, if $y(\xi)-\tilde{y}(\xi) \in \mathbb{Z}$ for all lattice sites $\xi$, then $E(y ; \tilde{y})=0$. This invariance of the energy is the primary source of analytical difficulties.

We call a deformation $y$ which minimises $E(y+u ; y)$ amongst all finite energy perturbations $u$ a globally stable equilibrium.

Building on [4] and the differential displacement maps first employed in [35], in \$2.2 we present a method by which we can identify dislocation cores and assign them a corresponding Burgers vector.

Our main result, Theorem 3.2 , states that there exists a globally stable equilibrium containing one geometrically necessary dislocation core.

The proof of this result is developed throughout the remainder of the paper. In $\$ 4$ we show that $\mathcal{E}(u):=E(\hat{y}+u ; \hat{y})$, where $\hat{y}$ is the linearised elasticity displacement field, is well-defined on a discrete $H^{1}$-function space describing finite energy states. In Theorem 4.5 we reformulate and refine Theorem 3.2 as a variational problem, by stating the existence of a global minimiser of $\mathcal{E}$ in that space. $\$ 5$ is then devoted to the proof of Theorem 4.5 .

Further, in $\$ 3$ we discuss briefly how to expoit the global stability result of Theorem 4.5 to construct locally stable configurations containing finitely many dislocation cores or configurations with dislocations in a domain with boundary.

\section{Geometric and Topological Preliminaries}

2.1. Anti-plane displacements of the BCC lattice. Although our analysis can be applied in other situations, it will be notationally convenient and physically relevant to restrict our attention to the body-centred cubic (BCC) lattice, which may be defined by

$$
\mathcal{L}:=\mathrm{BZ}^{3} \quad \text { where } \quad \mathrm{B}:=s\left[\begin{array}{ccc}
\sqrt{8 / 9} & \sqrt{2 / 9} & 0 \\
0 & \sqrt{2 / 3} & 0 \\
1 / 3 & -1 / 3 & 1
\end{array}\right],
$$


and $s>0$ is a scaling factor that we leave undefined for now.

To define anti-plane displacements, we fix the lattice vector $\nu:=[0,0, s]$ and define the projection

$$
\Pi_{\nu}:=\mathrm{I}-\frac{\nu}{|\nu|} \otimes \frac{\nu}{|\nu|},
$$

which 'flattens' the Bravais lattice $\mathcal{L}$ onto the lattice plane with normal $\nu$; see Figure $1(\mathrm{a})$. It is straightforward to check that the set $\Pi_{\nu}(\mathcal{L})$ is a two-dimensional triangular lattice embedded in $\mathbb{R}^{3}$, with lattice constant $s \sqrt{8 / 9}$; we will choose $s=\sqrt{9 / 8}$ so that the planar lattice constant is 1 . We further shift the origin so that the projected set may be identified with

$$
\Lambda:=\left(\frac{1}{2}, \frac{\sqrt{3}}{6}\right)^{T}+\left[a_{1}, a_{2}\right] \cdot \mathbb{Z}^{2}, \quad \text { where } a_{1}=(1,0)^{T} \text { and } a_{2}=\left(\frac{1}{2}, \frac{\sqrt{3}}{2}\right)^{T} .
$$

An anti-plane displacement in the direction $\nu$ (or, simply, displacement), is a map $y: \Lambda \rightarrow$ $\mathbb{R}$. The set of all displacements is denoted by $\mathscr{W}$. A displacement $y$ gives rise to a lattice deformation $Y: \mathcal{L} \rightarrow \mathbb{R}^{3}$,

$$
Y(\eta):=\eta+y\left(\left(\frac{1}{2}, \frac{\sqrt{3}}{6}\right)^{T}+\Pi_{\nu} \eta\right) \nu, \quad \eta \in \mathcal{L} .
$$

Let $y, \tilde{y}$ be displacements and $Y, \tilde{Y}$ the associated deformations. We say that $y, \tilde{y}$ are equivalent if $Y(\mathcal{L})=\tilde{Y}(\mathcal{L})$ (i.e., they describe the same atomistic configurations). It is easy to see that $y, \tilde{y}$ are equivalent if and only if $(y-\tilde{y})(\Lambda) \subseteq \mathbb{Z}$.

2.2. Bonds and bond lengths. Each $\xi \in \Lambda$ has six nearest neighbours, $\xi+a_{i}, i=1, \ldots, 6$, where $a_{1}=(1,0)^{T}$ and $a_{i}=\mathrm{R}_{6}^{i-1} a_{1}$ where $\mathrm{R}_{6}$ denotes a rotation through angle $\pi / 3$. At a point $\xi \in \Lambda$, we define the set of outward-pointing nearest neighbour bonds

$$
\mathcal{R}_{\xi}:=\left\{\left(\xi, \xi+a_{i}\right) \mid i=1, \ldots, 6\right\}=\{(\xi, \eta)|\eta \in \Lambda,| \xi-\eta \mid=1\},
$$

and furthermore define the set of all bonds to be the union

$$
\mathcal{B}:=\bigcup_{\xi \in \Lambda} \mathcal{R}_{\xi}=\left\{\left(\xi, \xi+a_{i}\right) \mid \xi \in \Lambda, i=1, \ldots, 6\right\}=\left\{(\xi, \eta) \in \Lambda^{2}|| \xi-\eta \mid=1\right\} .
$$

For any bond $b=\left(\xi, \xi+a_{i}\right)$, we denote the reverse bond by $-b:=\left(\xi+a_{i}, \xi\right)$.

For $b=(\xi, \eta) \in \mathcal{B}$ we define the difference operator

$$
D y_{b}:=y(\eta)-y(\xi) \text {. }
$$

Moreover, we set $D y:=\left(D y_{b}\right)_{b \in \mathcal{B}}$. We also note that $D y_{-b}=-D y_{b}$.

With this notation, we can now define two important discrete function spaces: fixing a reference lattice point $\xi_{0}=\left(0, \frac{\sqrt{3}}{3}\right)^{T}$,

$$
\begin{aligned}
\mathscr{W}_{0} & :=\left\{v \in \mathscr{W} \mid v\left(\xi_{0}\right)=0 \text { and } \operatorname{supp}(D v) \text { is bounded }\right\}, \quad \text { and } \\
\dot{\mathscr{W}}^{1,2} & :=\left\{v \in \mathscr{W} \mid v\left(\xi_{0}\right)=0 \text { and } D v \in \ell^{2}(\mathcal{B})\right\} .
\end{aligned}
$$

It is shown in [24, Prop. 9] that $\dot{\mathscr{W}}^{1,2}$ is a Hilbert space and $\mathscr{W}_{0} \subset \dot{\mathscr{W}}^{1,2}$ is dense.

We now introduce a crucial concept required to define the notation of dislocation. We denote the set of bond length 1-forms by

$$
[D y]:=\left\{\alpha: \mathcal{B} \rightarrow[-1 / 2,1 / 2] \mid \alpha_{-b}=\alpha_{b} \text { and } D y_{b}-\alpha_{b} \in \mathbb{Z} \text { for all } b \in \mathcal{B}\right\} .
$$

We note that, if $D y_{b} \notin \frac{1}{2}+\mathbb{Z}$ for all $b \in \mathcal{B}$, then $\alpha \in[D y]$ is unique, but in general there is ambiguity in the definition of $\alpha$. This non-uniqueness is an issue which we will return to in $\S 2.5$.

The motivation behind this definition is that $\alpha_{b}$ defines the 'shortest bond length' between the two lines of nuclei represented by the $2 \mathrm{D}$ lattice sites $\xi, \xi^{\prime}$, where $b=\left(\xi, \xi^{\prime}\right)$, in that

$$
\min _{\substack{\eta, \eta^{\prime} \in \mathcal{L} \\ \Pi_{\nu} \eta=\xi, \Pi_{\nu} \eta^{\prime}=\xi^{\prime}}}\left|y(\eta)-y\left(\eta^{\prime}\right)\right|=\sqrt{1+\alpha_{b}^{2}} ;
$$




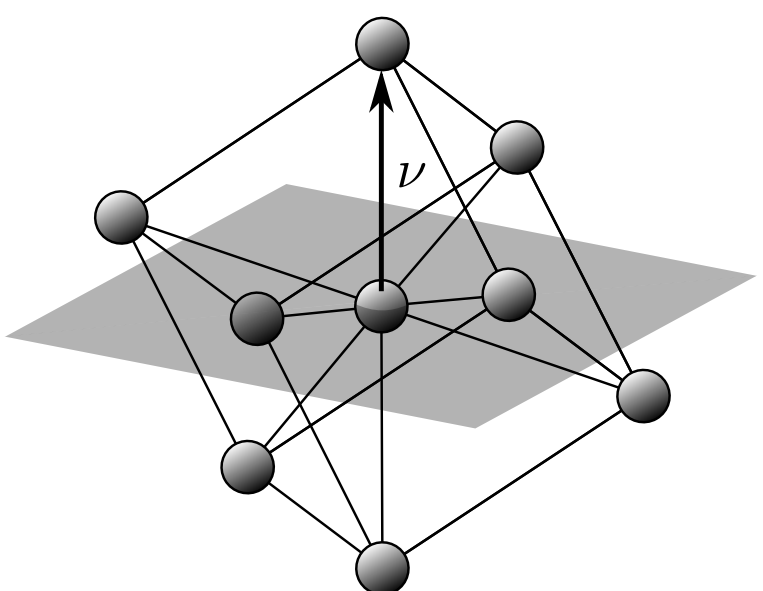

(a) Part of $\mathcal{L}$, showing the BCC unit cell, $\nu$ and the plane perpendicular to it.

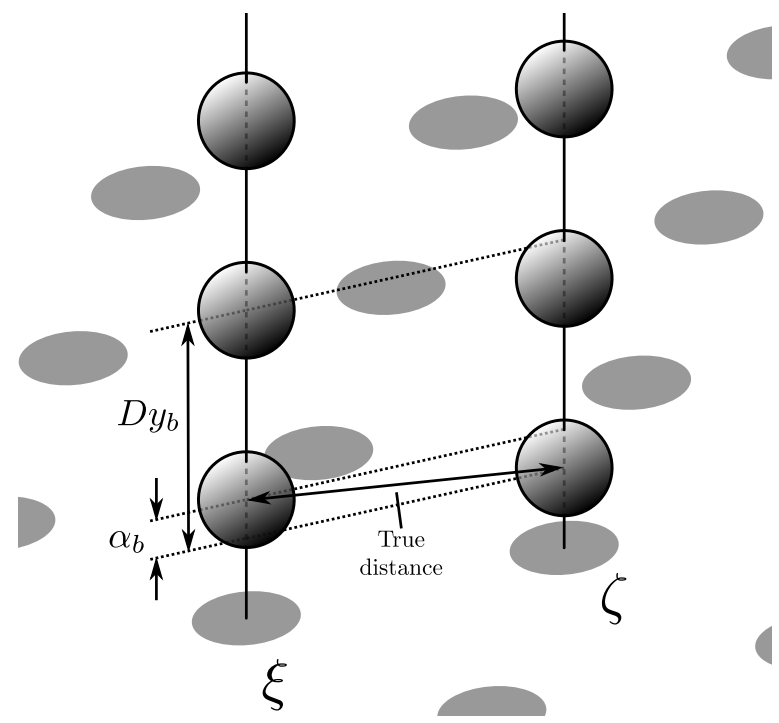

(b) An illustration of the definition of $\alpha_{b}$ and its relationship to the shortest distance between atoms.

FIGURE 1. Illustrations of the lattice geometry.

see also Figure $1(\mathrm{~b})$

The importance of the concept of bond length stems from the fact that, due to the invariance of the lattice under adding integer shifts to a displacement, the energy of the lattice can only depend on $\alpha_{b}$, but not on $D y_{b}$ directly.

2.3. The lattice complex. In this section, we review some terminology of discrete algebraic topology which is convenient for our analysis. We follow the language described in [4], where more details and applications to the study of dislocations can be found.

Repeating the definitions of $\Lambda, \mathcal{B}$, we define a lattice complex as in [4, §2.3.3], with

$$
\begin{aligned}
& \Lambda:=\left\{\xi \in \mathbb{R}^{2} \mid \xi \in \Pi_{\nu} \mathcal{L}+\left(\frac{1}{2}, \frac{\sqrt{3}}{6}\right)^{T}\right\}, \\
& \mathcal{B}:=\left\{(\xi, \zeta) \in \Lambda^{2}|| \xi-\zeta \mid=1\right\}, \quad \text { and } \\
& \mathcal{C}:=\left\{(\xi, \zeta, \eta) \in \Lambda^{3} \mid(\xi, \zeta),(\zeta, \eta),(\eta, \xi) \in \mathcal{B}\right\},
\end{aligned}
$$

denoting, respectively, the sets of 0-cells, 1-cells and 2-cells of the lattice complex respectively (see Figure 2 for an illustration). From now on, we will not explicitly use the terms $p$-cell, $p$-chain and $p$-cochain as defined in [4, §2.2], preferring instead the more evocative terminology 'lattice points' for elements of $\Lambda$, 'bonds' for elements of $\mathcal{B}$, and 'cells' for elements of $\mathcal{C}$. We note the additive structure that may be defined on these objects, and write $a \in A$ to mean that $a$ is an elementary $p$-cell contained in the $p$-chain $A$. We also frequently use the boundary operator $\partial$, which maps $p$-chains to their boundaries, assigning orientations in the usual way.

We then follow [4, §3] in defining $p$-forms and integration on the lattice, writing

$$
\int_{U} F:=\sum_{e \in U} F(e)
$$

where $U$ is a $p$-chain, $e$ are $p$-cells, and $F$ is a $p$-form (i.e. a real-valued function on $p$-cells). We note that this definition is linear in $F$ and $U$, in the sense that

$$
\begin{aligned}
\int_{U+V} \lambda F+G & =\lambda \sum_{e \in U} F(e)+\lambda \sum_{e \in V} F(e)+\sum_{e \in U} G(e)+\sum_{e \in V} G(e), \\
& =\lambda \int_{U} F+\int_{U} G+\lambda \int_{V} F+\int_{V} G
\end{aligned}
$$




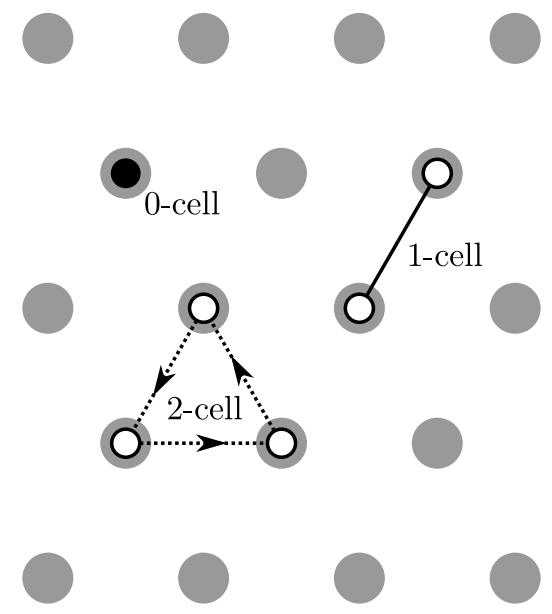

FiguRE 2. An illustration of 0-, 1- and 2-cells in the triangular lattice. The arrows show the boundary of the positively-oriented 2-cell. Note that orientation only makes sense for 1 - and 2-cells.

for any $\lambda \in \mathbb{R}, p$-chains $U$ and $V$, and $p$-forms $F$ and $G$. We remark here that 'bond length 1 -forms' $\alpha$ as defined in $\$ 2.2$ are true 1-forms in the sense defined in [4, §3.1].

Finally, we define paths in the natural way as 1-chains

$$
\Gamma:=\sum_{k=1}^{L}\left(\xi_{k}, \xi_{k+1}\right),
$$

where $\left(\xi_{k}, \xi_{k+1}\right) \in \mathcal{B}$ for each $k$, and we denote the length of a path $\Gamma$ by $|\Gamma|:=L$.

2.4. Measures of Lattice Distance. Since we will make use of more than simply the algebraic structure that a lattice complex entails, we will occasionally abuse the notation given above by identifying bonds and cells with their closed convex hulls; that is, we write

$$
\begin{aligned}
& x \in b=(\xi, \zeta) \quad \text { to mean } \quad x \in \operatorname{conv}\{\xi, \zeta\}, \quad \text { and } \\
& x \in C=(\xi, \zeta, \eta) \text { to mean } x \in \operatorname{conv}\{\xi, \zeta, \eta\} \text {, }
\end{aligned}
$$

where $\operatorname{conv}(\Omega)$ denotes the closed convex hull of a set $\Omega \subset \mathbb{R}^{2}$; it will be clear from the context whether we are referring to spatial points or subchains. Since we frequently refer to them, we define $x^{C}$ to be the barycentre of a cell $C$, and $C_{0}$ the cell for which $x^{C_{0}}=0$.

Using this form of the notation, we define the distance from each kind of cell to the origin as

$$
\begin{aligned}
d_{\xi} & :=|\xi|, \\
d_{b} & :=\inf _{x \in b}|x|, \\
d_{C} & :=\inf _{x \in C}|x|,
\end{aligned}
$$

which corresponds to the usual notion of distance between sets in Euclidean space.

The second notion of distance we will use is the graph theoretic notion. Since $\Lambda$ can be identified with a planar graph with edges $b \in \mathcal{B}$, we can further identify cells with nodes in the dual graph, and bonds as edges in this graph (see [9, §4.6]). This allows us to define the hopping distance, $\operatorname{hop}_{2}\left(C, C^{\prime}\right)$ as the length of the shortest path in the dual graph between the cells $C, C^{\prime} \in \mathcal{C}$, as in [9, §1.3]. We note that since the dual graph is connected, this distance is always finite, and we have the following 'triangle inequality' for any dual lattice points $A, B$ and $C$ :

$$
\operatorname{hop}_{2}(A, C) \leq \operatorname{hop}_{2}(A, B)+\operatorname{hop}_{2}(B, C) \text {. }
$$




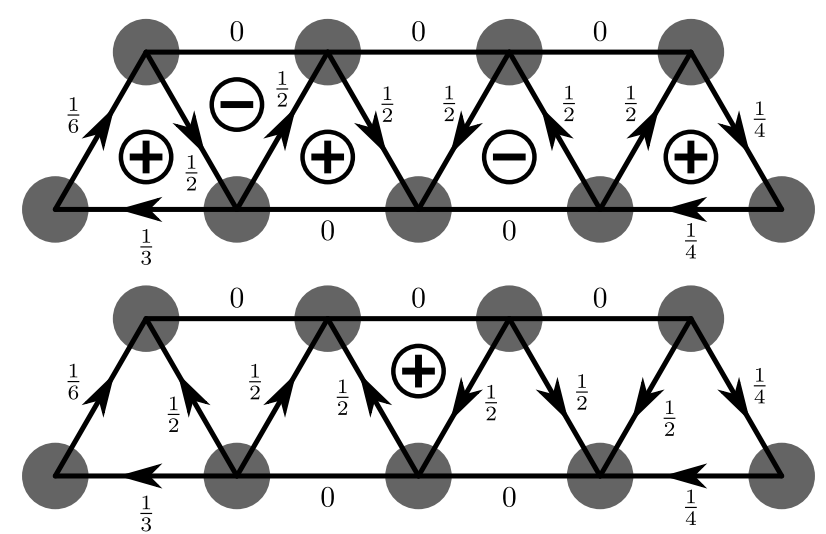

FiguRE 3. Two examples of bond length 1-forms corresponding to the same deformation. The numbers are the value of the 1-form on the relevant bond, and arrows indicate the bond direction in which it is positive. Note the number and positions of the dislocation cores present change, but the sum of the Burgers vectors does not.

2.5. The Burgers vector. We now define the notion of Burgers vector we use in our model, which is a fundamental geometric concept describing the nature of a dislocation [15].

We call a path $\Gamma=\sum_{k=1}^{L}\left(\xi_{k}, \xi_{k+1}\right)$ as defined in 2.2) a Burgers loop (or, simply, loop) if $\xi_{1}=\xi_{L+1}$ (or equivalently $\partial_{1} \Gamma=0$; this implies $\Gamma=\partial_{2} A$ for some sum of cells $A$, since the lattice complex is perfect [4, §2.2.1, Axiom (A4)]). If $\Gamma$ is a loop, $y \in \mathscr{W}$, and $\alpha \in[D y]$ an associated bondlength 1-form, then

$$
\int_{\Gamma} \alpha=\sum_{b \in \Gamma} D y_{b}+\sum_{b \in \Gamma}\left(\alpha_{b}-D y_{b}\right)=0+N \in \mathbb{Z} .
$$

We call the integer $N$ the Burgers vector of the bond length 1-form $\alpha$ around the loop $\Gamma$.

Definition 1 (Dislocation Core). A dislocation core of a bond length 1-form $\alpha$ is a positively oriented 2-cell $C$ such that $\int_{\partial C} \alpha \neq 0$.

We will refer to cores as being 'contained in' $\alpha$. For future reference, we remark that

$$
\left|\int_{\partial C} \alpha\right| \leq \frac{|\partial C|}{2}=\frac{3}{2}
$$

that is, the Burgers vector around a single 2-cell can only be $-1,0$ or 1 , and hence we define the sets of dislocation cores

$$
\begin{aligned}
& \mathcal{C}^{+}[\alpha]:=\left\{C \in \mathcal{C} \mid C \text { positively oriented, } \int_{\partial C} \alpha=+1\right\} \\
& \mathcal{C}^{-}[\alpha]:=\left\{C \in \mathcal{C} \mid C \text { positively oriented, } \int_{\partial C} \alpha=-1\right\} \\
& \mathcal{C}^{ \pm}[\alpha]:=\mathcal{C}^{+}[\alpha] \cup \mathcal{C}^{-}[\alpha] .
\end{aligned}
$$

Remark 2.1. It is interesting to note that if $\alpha, \alpha^{\prime} \in[D y]$, then they need not have the same number of cores; see Figure 3 for an illustration of this fact.

The only point at which this ambiguity is an issue is if $\alpha$ has $C, C^{\prime} \in \mathcal{C}^{ \pm}[\alpha]$ which are adjacent. In that case, it may be checked that the $b \in \partial C$ such that $-b \in \partial C^{\prime}$ must have $\alpha_{b} \in\{-1 / 2,0,1 / 2\}$. In the case where $\alpha_{b}= \pm 1 / 2$, redefining $\alpha_{b}=\mp 1 / 2$ removes these cores, and $\alpha$ remains a bond length 1 -form in $[D y]$, so we will always assume that minimising sequences have $\alpha_{b}=0$ for any bond $b$ shared by 2 adjacent cores. 
The net Burgers vector is obtained by summing the signs of the cores, or equivalently, by computing the Burgers vector on a sufficiently large loop enclosing all cores. Since $\alpha \in[D y]$ is not necessarily unique for a given $y$, we ensure that such a concept can be defined unambiguously.

For our purposes it will be enough to consider displacements with some prescribed far-field behaviour.

Proposition 2.2. Let $y \in \mathscr{W}$ and $\alpha \in[D y]$ such that $\alpha_{b} \rightarrow 0$ as $d_{b} \rightarrow \infty$. Then, for any $\alpha^{\prime} \in[D y], \mathcal{C}^{ \pm}\left[\alpha^{\prime}\right]$ is finite and

$$
\sum_{C \in \mathcal{C}^{ \pm}\left[\alpha^{\prime}\right]} \int_{\partial C} \alpha^{\prime}=\sum_{C \in \mathcal{C}^{ \pm}[\alpha]} \int_{\partial C} \alpha=\int_{\Gamma} \alpha,
$$

where $\Gamma$ is any loop that encloses all cores in $\alpha$.

Proof. If $\alpha_{b} \rightarrow 0$ as $d_{b} \rightarrow \infty$ then

$$
\int_{\partial C} \alpha \rightarrow 0 \quad \text { as } d_{C} \rightarrow \infty .
$$

Since $\int_{\partial C} \alpha \in \mathbb{Z}$ it follows that $\int_{\partial C} \alpha=0$ for $d_{C}$ sufficiently large, and hence the number of dislocation cores present in $\alpha$ is finite.

Moreover, since $\alpha_{b} \in(-1 / 2,1 / 2)$ for $d_{b}$ sufficiently large, it follows that $\alpha_{b}=\alpha_{b}^{\prime}$ for all $\alpha^{\prime} \in[D y]$ and $d_{b}$ sufficiently large. In particular, $\mathcal{C}^{ \pm}\left[\alpha^{\prime}\right]$ is also finite.

To prove (2.5), let $\Gamma$ be a loop that encloses all the cores in $\alpha$ for which $\Gamma=\partial A$. Then

$$
\int_{\Gamma} \alpha=\sum_{C \in A} \int_{\partial C} \alpha=\sum_{C \in \mathcal{C}^{ \pm}} \int_{\partial C} \alpha .
$$

Taking $\Gamma$ such that $D y_{b}=\alpha_{b}=\alpha_{b}^{\prime}$ for all $b \in \Gamma$ we obtain the first identity in 2.5) as well.

We can now formally define the net Burgers vector.

Definition 2 (Net Burgers Vector). Let $y \in \mathscr{W}$ such that $\alpha_{b} \rightarrow 0$ as $d_{b} \rightarrow \infty$ for some $\alpha \in[D y]$. Then we define the net Burgers vector of $y$ to be

$$
B[y]:=\sum_{C \in \mathcal{C}^{ \pm}[\alpha]} \int_{\partial C} \alpha,
$$

for an arbitrary $\alpha \in[D y]$.

The quantity $B[y]$ can be experimentally observed from outside the system, by determining the strain at 'infinity'. For example, if $B[y]=1$, then this tells the observer that there must be at least one dislocation in the system, but nothing about the total number.

\section{MAin Result}

In this section we present the main result of the paper with accompanying assumptions.

3.1. Energy difference functional. Before we can state the main result, we introduce another key concept that we employ in its formulation and proof: the energy difference functional. We assume that lattice sites (corresponding to lines of atoms in the BCC crystal) interact via a nearest-neighbour pair potential $\psi \in C(\mathbb{R}) \cap C^{4}(\mathbb{R} \backslash(\mathbb{Z}+1 / 2))$, which satisfies the following properties: 
$(\psi 1) \psi$ is 1 -periodic;

$(\psi 2) \psi$ and $\psi\left(\frac{1}{2}+\cdot\right)$ are even;

$(\psi 3) \psi(r)=0$ if and only if $r \in \mathbb{Z}$;

$(\psi 4) \psi^{\prime \prime}(0)=\mu>0$.

$(\psi 5) \psi(x) \geq \frac{1}{2} \psi^{\prime \prime}(0) x^{2}$ for all $x \in\left[-\frac{1}{2}, \frac{1}{2}\right]$.

Remark 3.1. Assumptions $(\psi 1)-(\psi 4)$ are very general, and are natural in the physical context: $(\psi 1)$ and $(\psi 2)$ encode lattice symmetries, while $(\psi 3)$ and $(\psi 4)$ state that the system has a stable crystalline ground state.

The only "technical" assumption is $(\psi 5)$. The reason for this assumption will become apparent in $\$ 5.1$, where we use it to establish an a priori bound on the number of dislocation dipoles in finite energy configurations. We believe that $(\psi 5)$ can be replaced with weaker variants, but cannot be removed altogether.

We remark that the requirement that $\psi \in \mathrm{C}^{4}(\mathbb{R} \backslash(\mathbb{Z}+1 / 2))$ can be relaxed further by modifying the proofs we give below, but since this adds little at the expense of readability, we omit such arguments here. The prototypical example of a potential satisfying $(\psi 1)-(\psi 5)$ is $\psi(r)=\psi^{\operatorname{lin}}(r):=\frac{1}{2} \operatorname{dist}(r, \mathbb{Z})^{2}$.

For two displacements $y, \tilde{y} \in \mathscr{W}$ we define the energy difference functional, formally for the moment, as

$$
E(y ; \tilde{y}):=\sum_{b \in \mathcal{B}}\left[\psi\left(D y_{b}\right)-\psi\left(D \tilde{y}_{b}\right)\right] .
$$

For example, if $y-\tilde{y} \in \mathscr{W}_{0}$, then $E(y ; \tilde{y})$ is clearly well-defined since the sum is effectively finite. For arbitrary displacements $y, \tilde{y}, E(y ; \tilde{y})$ need not be well-defined. However, we will show in $\$ 4.1$ that $E$ can, under certain conditions, be extended by continuity to relative displacements $y-\tilde{y} \in \dot{\mathscr{W}}^{1,2}$.

Using the terminology of energy differences, we can define what we mean by a stable equilibrium displacement. Intuitively, the definition entails that finite energy perturbations cannot lower the energy.

Definition 3 (Stable Equilibrium). A displacement $y \in \mathscr{W}$ is a locally stable equilibrium if there exists $\epsilon>0$ such that $E(y+u ; y) \geq 0$ for all $u \in \mathscr{W}_{0}$ with $\|D u\|_{2} \leq \epsilon$.

$A$ displacement $y \in \mathscr{W}$ is a globally stable equilibrium if $E(y+u ; y) \geq 0$ for all $u \in \mathscr{W}_{0}$.

3.2. Statement of the main result. Recalling Definitions 2 and 3 the existence of a screw dislocation can be formulated as follows.

Theorem 3.2 (Existence of a geometrically necessary dislocation). There exists a globally stable equilibrium displacement $y \in \mathscr{W}$ with net Burgers vector $B[y]=1$.

The notion of global stability described in Definition 3 is equivalent to the statement that a displacement is stable if any finite energy perturbation increases the energy of the system. Describing a dislocation configuration as the minimiser of an energy difference functional gives us access to the Direct Method of the Calculus of Variations.

We refer to this result as the existence of a 'geometrically necessary' dislocation since we do not prescribe the absolute number of dislocation cores, but only the net Burgers vector.

Outline of the proof of Theorem 3.2.

(1) We define a reference configuration $\hat{y}(\xi)=\frac{1}{2 \pi} \arctan \left(\frac{\xi_{2}}{\xi_{1}}\right)$ (the continuum linear elasticity solution for a dislocation), with the aim to minimise the energy difference functional $\mathcal{E}(u):=E(\hat{y}+u ; \hat{y})$ over a suitable class of functions $u$.

We show that this functional, initially defined over $\mathscr{W}_{0}$, can be continuously extended to a functional over $\dot{\mathscr{W}}^{1,2}$. 
(2) In order to use the Direct Method to establish the existence of a minimiser to $\mathcal{E}$, the crucial step is to obtain a global lower bound on the energy. This is the main step in the proof, and requires careful geometric estimates based on the number of dislocation cores and the distance between them. We shall prove that $\mathcal{E}(u) \gtrsim\|\beta\|_{2}^{2}-1$, where $\beta$ can be thought of as belonging to $[D u]$ (however, see (5.1) for the precise definition).

(3) This lower bound guarantees in particular that the number of dislocation cores is bounded along a minimising sequence as well as weak compactness of a minimising sequence $u^{n}$.

(4) The final step is to ensure that $\lim u^{n}$ has non-zero net-Burgers vector. This need not be the case since weak convergence of $D u^{n}$ allows for energy to be translated to infinity. In our present context it is possible, by introducing a dislocation dipole, to effectively translate the geometrically necessary core to infinity, and thus obtain a limiting displacement with zero net Burgers vector. We shift the minimising sequence and employ a concentration compactness argument to prevent this.

3.3. Locally stable equilibria. Theorem 3.2 establishes the existence of a configuration $y=$ $\hat{y}+u$, which is a globally stable equilibrium configuration for a single screw dislocation in an infinite lattice. From this starting point, it is possible to construct more general locally stable equilibrium configurations. The idea is (1) to superimpose copies of $y$ and define

$$
\tilde{z}(\xi):=\sum_{j=1}^{J} s_{j} y\left(\xi-x^{C_{j}}\right),
$$

where $C_{j} \in \mathcal{C}$ are cores in $\tilde{z}$ and $s_{j} \in\{ \pm 1\}$ the Burgers' vectors of these cores; (2) to show that $\tilde{z}$ is an approximate equilibrium when the cores $C_{j}$ are sufficiently far from one another; and (3) to apply the inverse function theorem to establish the existence of an equilibrium $z$ close to $\tilde{z}$.

Here, we only state two results that we obtain by this strategy, but refer for their proofs to [17], where we present them in a more general context.

In step (3) of the strategy outlined above we require a discrete ellipticity condition (3.1), which can be established rigorously, for example, for a piecewise quadratic potential.

Lemma 3.3 (Discrete ellipticity). Let $\psi(r):=\psi_{\operatorname{lin}}(r):=\frac{\lambda}{2} \operatorname{dist}(r, \mathbb{Z})^{2}$ and let $y=\hat{y}+u$, $u \in \dot{\mathscr{W}}^{1,2}$, be a locally stable equilibrium configuration. Then Dyb $\in \mathbb{R} \backslash\left(\frac{1}{2}+\mathbb{Z}\right)$ for all $b \in \mathcal{B}$, and hence

$$
\sum_{b \in \mathcal{B}} \psi^{\prime \prime}\left(D y_{b}\right) D v_{b}^{2} \geq \lambda\|D v\|_{2}^{2} \quad \forall v \in \dot{\mathscr{W}}^{1,2}
$$

Proof. Suppose there exists a bond $b=(\xi, \zeta)$ such that $D y_{b} \in \frac{1}{2}+\mathbb{Z}$. Let $z_{t}(\eta):=y+t \delta_{\eta, \xi}$, then a direct calculation shows that $E\left(z_{t} ; y\right)<0$ for some sufficiently small $t$ (either positive or negative).

The two results we state in the following admit general $\psi$, but require (3.1) as an assumption:

(STAB): There exists a locally stable equilibrium $y=\hat{y}+u, u \in \dot{\mathscr{W}}^{1,2}$, satisfying the ellipticity condition (3.1). Moreover, let $A$ be a finite union of cells such that $\mathcal{C}^{ \pm}[\alpha] \subset A$, for any $\alpha \in[D y]$.

Our first local stability result states that any configuration of dislocations is stable provided that the cores are sufficiently separated. In particular, it shows that there exist stable configurations with arbitrary net Burgers.

Corollary 3.4 (Finitely many cores). Suppose that (STAB) holds. 
Let $C_{j} \in \mathcal{C}, j=1, \ldots J, J \in \mathbb{N}$, be a finite collection of cells, and let $s_{j} \in\{ \pm 1\}$. There is a minimal separation distance $L_{0}>0$ such that, if $\min _{i \neq j}\left|x^{C_{j}}-x^{C_{i}}\right| \geq L_{0}$, then there exists a locally stable configuration $z \in \mathscr{W}$ such that, for any $\alpha \in[D z]$,

$$
\begin{aligned}
& \mathcal{C}^{ \pm}[\alpha] \subset \bigcup_{j=1}^{J}\left(x^{C_{j}}+A\right) \quad \text { and } \\
& \int_{\partial\left(x^{C_{j}}+A\right)} \alpha=s_{j}, \quad \text { for } j=1, \ldots, J .
\end{aligned}
$$

In particular, $B[z]=\sum_{j=1}^{J} s_{j}$.

Our second local stability result states that dislocations are stable provided they are sufficiently distant from any domain boundary. To state this result, let $\Omega:=\left\{\xi \in \Lambda \mid \xi_{2} \leq 0\right\}$ be a discrete half space and let $\mathcal{B}^{\Omega}:=\{b=(\xi, \zeta) \in \mathcal{B} \mid \xi, \zeta \in \Omega\}$ be the corresponding set of bonds.

Corollary 3.5 (Domain with boundary). Suppose that (STAB) holds.

Let $C \in \mathcal{C}$ such that $L:=-\left(x^{C}\right)_{2}>0$. If $L$ is sufficiently large, then there exists a locally stable half-space configuration $z: \Omega \rightarrow \mathbb{R}$ containing a dislocation. That is, for any $\alpha \in[D z]$,

(1) $\mathcal{C}^{ \pm}[\alpha] \subset x^{C}+A$,

(2) $\int_{\partial\left(x^{C}+A\right)} \alpha=1$, and

(3) there exists $\epsilon>0$ such that

$$
\sum_{b \in \mathcal{B}^{\Omega}}\left(\psi\left(D z_{b}+D v_{b}\right)-\psi\left(D z_{b}\right)\right)>0 \quad \forall v \in \mathscr{W}_{0},\|D v\|_{2}<\epsilon .
$$

3.4. Regularity. The globally stable equilibrium configuration $y$, whose existence we established in Theorem 3.2 is of the form $y=\hat{y}+u$, where $\hat{y}(\xi)=\frac{1}{2 \pi} \arctan \left(\frac{\xi_{2}}{\xi_{1}}\right)$ is the continuum linearised elasticity solution for a screw dislocation. We refer to $\$ 4$ and in particular to Theorem 4.5 for further details.

This fact implies that only a finite amount of energy is stored in the dislocation core, and that, up to some fixed prescribed error tolerance, the linearised elasticity displacement field is accurate outside of some fixed radius. These observations give rise to new points of view on the concepts of dislocation core energy and core radius, which we explore in [16]. In particular the core radius is an interesting concept related to the decay of the "corrector" $u$ to the configuration $\hat{y}$ predicted by linear elasticity. Here, we state a regularity result proven in more general form in [10], which precisely quantifies the rate of decay of $D u$. In effect, the results states that the decay of $D u$ is the same as predicted by linearised elasticity.

Proposition 3.6. Let $y=\hat{y}+u, u \in \dot{\mathscr{W}}^{1,2}$ be a locally stable equilibrium, then there exists $C$ such that

$$
\left|D u_{b}\right| \leq C d_{b}^{-2} \quad \forall b \in \mathcal{B}
$$

Remark 3.7. One may expect, and numerical simulations confirm this, that the corrector $u$ satisfies the three-fold symmetry of the lattice $\Lambda$ with respect to its origin (recall that the origin lies in the barycentre of a cell). Exploiting this symmetry, one can observe that the decay rate is in fact $\left|D u_{b}\right| \leq C d_{b}^{-4}$. However, as soon as the symmetry is broken, for example by applying a small shear displacement at infinity, or by moving the core off the centre of the cell, the generic rate $(3.2)$ is observed also numerically. 


\section{Analysis of the EnERGy Difference FunCtional}

4.1. Extension of the energy difference functional. We fix a displacement $\hat{y}$ and define the functional $\mathcal{E}(u):=E(\hat{y}+u ; \hat{y})$. For $u \in \mathscr{W}_{0}$ this is always well-defined. If $D \hat{y}_{b} \in \mathbb{R} \backslash(\mathbb{Z}+B(\epsilon))$ for all $b \in \mathcal{B}$, where $\epsilon>0$, then the first and second variations (in the sense of directional derivatives) are also well-defined, and given by

$$
\begin{aligned}
\langle\delta \mathcal{E}(0), v\rangle & =\sum_{b \in \mathcal{B}} \psi^{\prime}\left(D \hat{y}_{b}\right) \cdot D v_{b}, \quad \text { for } v \in \mathscr{W}_{0}, \quad \text { and } \\
\left\langle\delta^{2} \mathcal{E}(0) v, w\right\rangle & =\sum_{b \in \mathcal{B}} \psi^{\prime \prime}\left(D \hat{y}_{b}\right) \cdot D v_{b} D w_{b} \quad \text { for } v, w \in \mathscr{W}_{0} .
\end{aligned}
$$

$\delta^{2} \mathcal{E}(0)$ can clearly be extended by continuity to $v, w \in \dot{\mathscr{W}}^{1,2}$, but this is less obvious for $\delta \mathcal{E}(0)$ or for $\mathcal{E}$ itself. We first state a general result.

Lemma 4.1. Let $\hat{y} \in \mathscr{W}$ satisfy $D \hat{y}_{b} \in \mathbb{R} \backslash(\mathbb{Z}+B(\epsilon))$ for some $\epsilon>0$ and suppose that $\delta \mathcal{E}(0)$ is a bounded linear functional $\left(\langle\delta \mathcal{E}(0), v\rangle \leq C\|D v\|_{2}\right.$ for all $\left.v \in \mathscr{W}_{0}\right)$. Then, $\mathcal{E}: \mathscr{W}_{0} \rightarrow \mathbb{R}$ is continuous with respect to the norm $\|D \cdot\|_{2}$; hence, there exists a unique continuous extension of $\mathcal{E}$ to $\dot{\mathscr{W}}^{1,2}$.

Proof. The proof of this result is analogous to the proof of Theorem 2.8 (ii) in [26]. For convenience we give a brief outline.

For $u \in \mathscr{W}_{0}$ it is easy to see that

$$
\mathcal{E}(u)=\sum_{b \in \mathcal{B}}\left[\psi\left(D \hat{y}_{b}+D u_{b}\right)-\psi\left(D \hat{y}_{b}\right)-\psi^{\prime}\left(D \hat{y}_{b}\right) D u_{b}\right]+\sum_{b \in \mathcal{B}} \psi^{\prime}\left(D \hat{y}_{b}\right) D u_{b} .
$$

Since we assume that $\delta \mathcal{E}(0)$ is a bounded functional, the second term on the right-hand side is continuous. Using the fact that $\|D w\|_{\infty} \leq\|D w\|_{2}$, the smoothness of $\psi$, and the fact that each summand in the first group is effectively quadratic in $D u_{b}$, it is easy to show that the second term on the right-hand side is continous as well.

For future reference, we now derive a simple condition on $\hat{y}$ under which $\delta \mathcal{E}(0)$ is a bounded functional. Applying summation by parts to 4.1) we obtain

$$
\langle\delta \mathcal{E}(0), v\rangle=\sum_{\xi \in \Lambda} f(\xi) \cdot v(\xi), \quad \text { where } \quad f(\xi):=\sum_{b \in \mathcal{R}_{\xi}} \psi^{\prime}\left(D \hat{y}_{b}(\xi)\right)
$$

is the force acting on atom $\xi$ under the displacement $\hat{y}$. The following result states that, if $\hat{y}$ is sufficiently close to equilibrium in the far-field, then $\delta \mathcal{E}(0)$ is a bounded linear functional.

Lemma 4.2. Suppose that a displacement $\hat{y}$ has associated forces $f(\xi)$ satisfying the bound $|f(\xi)| \leq C_{1}(1+|\xi|)^{-t}$ for some $t>2$, then $\langle\delta \mathcal{E}(0), v\rangle \leq C_{2}\|D v\|_{2}$ for all $v \in \mathscr{W}_{0}$.

Proof. Proposition 12 in [24] immediately implies that

$$
\left\|\frac{v}{\log (|\xi|+2)}\right\|_{\infty} \leq C\|D v\|_{2}
$$

for some constant $C>0$. (This inequality is essentially a consequence of the embedding $\|v\|_{\mathrm{BMO}} \leq C\|\nabla v\|_{L^{2}}$ for $v \in C^{1}\left(\mathbb{R}^{2}\right)$.)

We can therefore estimate

$$
|\langle\delta \mathcal{E}(0), v\rangle| \leq \sum_{\xi \in \Lambda}|f(\xi)||v(\xi)| \leq\|\log (|\xi|+2) f\|_{1}\left\|\frac{v}{\log (|\xi|+2)}\right\|_{\infty} .
$$

The assumption $|f(\xi)| \leq C|\xi|^{-t}$ with $t>2$ implies that $\|\log (|\xi|+2) f\|_{1}$ is finite. 


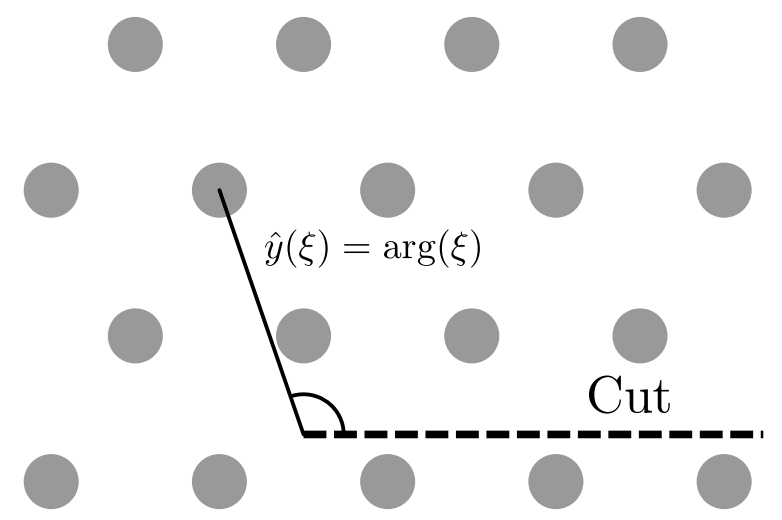

FiguRE 4. An illustration of the coordinate system and reference configuration chosen.

4.2. The reference displacement. We now specify the reference displacement $\hat{y}$ used in the definition of $\mathcal{E}$ in 4 4.1. It is best to think of $\hat{y}$ as prescribing a far-field boundary condition $y(\xi) \sim \hat{y}(\xi)$ as $|\xi| \rightarrow \infty$. We wish to choose $\hat{y}$ in such a way that it enforces a geometrically necessary dislocation, and at the same time satisfies the condition of Lemma 4.2 .

A natural choice is the dislocation displacement field from linear elasticity theory. Since it is instructive (though not essential to our proofs) we give a brief motivation of this construction. In the far-field, we expect that continuum linearized elasticity theory is a good approximation to the atomistic equilibrium condition $\delta \mathcal{E}(0)=0$. This can be formalized by first deriving the Cauchy-Born approximation and then linearising it. Due to the hexagonal symmetry of $\Lambda$ one finds that the linearised continuum approximation is simply Laplace's equation, $\Delta \hat{y}(x)=0$.

Hence, following Section 3-2 in Hirth \& Lothe [15], we define $\hat{y}$ as follows:

$$
\hat{y}(x):=\frac{1}{2 \pi} \arg (x)=\frac{1}{2 \pi} \arctan \left(\frac{x_{2}}{x_{1}}\right),
$$

where we identify $x \in \mathbb{R}^{2}$ with the point $x_{1}+i x_{2} \in \mathbb{C}$, and the branch cut is taken along the positive $\xi_{1}$-axis, as shown in Figure 4 .

The gradient (away from the branch cut) is given by

$$
\nabla \hat{y}(x)=\left(\frac{-x_{2}}{2 \pi r^{2}}, \frac{x_{1}}{2 \pi r^{2}}\right)^{T}
$$

where $r:=|x|$. This function can be extended to a function in $C^{\infty}\left(\mathbb{R}^{2} \backslash\{0\}\right)$, which we take as the definition of $\nabla \hat{y}$ from now on. Moreover, we can check that indeed $\Delta \hat{y}(x)=\operatorname{div}(\nabla \hat{y}(x))=0$, in the pointwise sense, for $x \neq 0$.

Let $\hat{\alpha}=\left(\hat{\alpha}_{b}\right)_{b \in \mathcal{B}}$ be a bond-length 1 -form associated with $\hat{y}$; we claim this is unique, and the following lemma provides a convenient formula for $\hat{\alpha}_{b}$ in terms of $\nabla \hat{y}$.

Lemma 4.3. Let $\hat{\alpha} \in[D \hat{y}]$ then for any bond $b=\left(\xi, \xi+a_{i}\right) \in \mathcal{B}$, we have

$$
\hat{\alpha}_{b}=\int_{0}^{1} \nabla \hat{y}\left(\xi+t a_{i}\right) \cdot a_{i} \mathrm{~d} t .
$$

Proof. By definition, $\nabla \hat{y}$ is independent of the choice of branch cut. Moreover, if the branch cut is chosen differently, then the displacement at each site is only changed by an integer, which means $\hat{\alpha}$ does not change; hence, $\hat{\alpha}$ is also independent of the branch cut.

Now fix $b=\left(\xi, \xi+a_{i}\right) \in \mathcal{B}$. Since the origin lies at the centre of a cell we can redefine $\hat{y}$ with a branch cut that does not intersect $b$. The Fundamental Theorem of Calculus gives

$$
\int_{0}^{1} \nabla \hat{y}\left(\xi+t a_{i}\right) \cdot a_{i} \mathrm{~d} t=\hat{y}\left(\xi+a_{i}\right)-\hat{y}(\xi)=\frac{1}{2 \pi}\left(\arg \left(\xi+a_{i}\right)-\arg (\xi)\right),
$$

and since we have assumed that $b$ is a nearest neighbour bond, it has length 1 . The term on the right hand side is $1 / 2 \pi$ times the angle formed by the points $\xi, 0$ and $\xi+a_{i}$, which is 
maximised by making $\xi$ and $\xi+a_{i}$ as close to the origin as possible - that is, when $\xi$ and $\xi+a_{i}$ are on the boundary of $C_{0}$. It follows that the angle can be no larger than $\frac{2 \pi}{3}$, and hence $\hat{\alpha}_{b}=\hat{y}\left(\xi+a_{i}\right)-\hat{y}(\xi) \in\left[-\frac{1}{3}, \frac{1}{3}\right]$. This implies that $\hat{\alpha}$ is unique, since $D \hat{y}_{b} \neq \pm \frac{1}{2}$ for all $b \in \mathcal{B}$.

As an immediate corollary of Lemma 4.3 we obtain the following bound on $\hat{\alpha}_{b}$ :

$$
\left|\hat{\alpha}_{b}\right| \leq \frac{1}{2 \pi d_{b}} \quad \forall b \in \mathcal{B}
$$

We conclude the analysis of $\hat{y}$ by showing that it satisfies the conditions of Lemma 4.2 .

Lemma 4.4. Let $\hat{y}$ be defined by 4.5), and let $f(\xi), \xi \in \Lambda$, be the associated forces (see (4.3)), then $\hat{\alpha} \in[D \hat{y}]$ satisfies $\hat{\alpha}_{b} \in[-1 / 3,1 / 3]$ and

$$
|f(\xi)| \lesssim|\xi|^{-3} \quad \forall \xi \in \Lambda
$$

In particular $\hat{y}$ satisfies all conditions of Lemma 4.2.

Proof. Recall from (4.3) that

$$
f(\xi)=\sum_{b \in \mathcal{R}(\xi)} \psi^{\prime}\left(D_{b} \hat{y}\right)=\sum_{b \in \mathcal{R}(\xi)} \psi^{\prime}\left(\hat{\alpha}_{b}\right)
$$

Taylor expanding $\psi_{b}^{\prime}$ to third order, using the fact that $\psi^{\prime}(0)=\psi^{\prime \prime \prime}(0)=0$ (since $\psi$ is even about 0 ), gives

$$
f(\xi)=\sum_{b \in \mathcal{R}(\xi)}\left[\psi^{\prime \prime}(0) \hat{\alpha}_{b}+\frac{1}{6} \psi^{(4)}\left(s_{b}\right)\left(\hat{\alpha}_{b}\right)^{3}\right],
$$

for some $s_{b} \in \operatorname{conv}\left\{0, \hat{\alpha}_{b}\right\}$. Applying 4.9 we obtain

$$
f(\xi)=\sum_{b \in \mathcal{R}(\xi)} \psi^{\prime \prime}(0) \hat{\alpha}_{b}+O\left(d_{b}^{-3}\right)
$$

We now inspect the sum on the right-hand side of 4.10 in more detail. Applying (4.7) we rewrite this sum as

$$
\sum_{b \in \mathcal{R}(\xi)} \psi^{\prime \prime}(0) \hat{\alpha}_{b}=\sum_{i=1}^{6} \psi^{\prime \prime}(0) \int_{0}^{1} \nabla \hat{y}\left(\xi+t a_{i}\right) \cdot a_{i} \mathrm{~d} t .
$$

Taylor expanding $\nabla \hat{y}\left(\xi+t a_{i}\right)$ and using the fact that $\left|\nabla^{4} \hat{y}(x)\right| \lesssim|x|^{-4}$, we obtain

$$
\int_{0}^{1} \nabla \hat{y}\left(\xi+t a_{i}\right) \cdot a_{i} \mathrm{~d} t=\nabla \hat{y}(\xi) \cdot a_{i}+\frac{1}{2} \nabla^{2} \hat{y}(\xi)\left[a_{i}, a_{i}\right]+\frac{1}{6} \nabla^{3} \hat{y}(\xi)\left[a_{i}, a_{i}, a_{i}\right]+O\left(|\xi|^{-4}\right) .
$$

Summing over $i=1, \ldots, 6$ the first and third terms cancel since $a_{i+3}=-a_{i}$, hence we obtain

$$
\sum_{b \in \mathcal{R}(\xi)} \psi^{\prime \prime}(0) \hat{\alpha}_{b}=\frac{1}{2} \sum_{i=1}^{6} a_{i}^{T} \nabla^{2} \hat{y}(\xi) a_{i}+O\left(|\xi|^{-4}\right) .
$$

We now observe that

$$
\frac{1}{2} \sum_{i=1}^{6} a_{i}^{T} \nabla^{2} \hat{y}(\xi) a_{i}=-\frac{3}{2} \Delta \hat{y}(\xi)=0 .
$$

Inserting the last identity into 4.11 and combining the resulting estimate with 4.10 we obtain the stated estimate on $|f(\xi)|$. 
4.3. The variational problem in $\dot{\mathscr{W}}^{1,2}$. Combining Lemma 4.4 with Lemma 4.1 and Lemma 4.2 , we deduce that $\mathcal{E}(u):=E(\hat{y}+u ; \hat{y})$ is a well-defined and continuous functional on $\dot{\mathscr{W}}^{1,2}$, where $\hat{y}$ is the reference configuration defined in (4.5). It will later be convenient to recall from the proof of Lemma 4.1 that the explicit definition of the extension is

$$
\mathcal{E}(u)=\sum_{b \in \mathcal{B}}\left[\psi\left(\hat{\alpha}_{b}+D u_{b}\right)-\psi\left(\hat{\alpha}_{b}\right)-\psi^{\prime}\left(\hat{\alpha}_{b}\right) D u_{b}\right]+\langle\delta \mathcal{E}(0), u\rangle
$$

In the next section, $\S 5$, we will prove the following result:

Theorem 4.5. There exists $u \in \dot{\mathscr{W}}^{1,2}$ such that $\mathcal{E}(u) \leq \mathcal{E}(v)$ for all $v \in \dot{\mathscr{W}}^{1,2}$.

As an immediate corollary we can now prove Theorem 3.2

Proof of Theorem 3.2. Let $y:=\hat{y}+u$, where $u$ is a minimizer of $\mathcal{E}$ in $\dot{\mathscr{W}}^{1,2}$. Since $D u \in \ell^{2}(\mathcal{B})$ it follows that $\left|D u_{b}\right| \rightarrow 0$ uniformly as $d_{b} \rightarrow \infty$. Using also the fact that $\left|\hat{\alpha}_{b}\right| \rightarrow 0$ uniformly (cf. (4.9)), we conclude that $\alpha_{b}=\hat{\alpha}_{b}+D u_{b}+z_{b}$, where $z_{b}$ is a compactly supported, integer-valued 1-form. From the definition of the net Burgers vector and from 2.5), it now follows immediately that $B[y]=B[\hat{y}]=1$. Moreover, minimality of $u$ implies that $y$ is a globally stable equilibrium in the sense of Definition 3 .

Theorem 4.5 is interesting in its own right: it shows that atomistic configurations containing dislocations can be obtained as global minimizers of a variational problem formulated over $\Lambda$. This is particularly useful for further study (e.g., of regularity; cf. § 3.4) of dislocations in this model.

We also remark that any local minimizer $u$ of $\mathcal{E}$ in $\dot{\mathscr{W}}^{1,2}$ would give rise to a locally stable equilibrium with net Burgers vector $B(\hat{y}+u)=1$. The advantage of local minimisers is that they can be computed numerically.

\section{Proof of Theorem 4.5}

As currently formulated, it is not obvious that the energy $\mathcal{E}$ is bounded below, and it is even less clear whether $\mathcal{E}$ is coercive in a sense which would allow us to invoke the Direct Method. This is due in large part to the fact that the reference configuration is nonlinear and $\psi$ is periodic, so the integrand has infinitely many energy wells.

The periodicity of $\psi$ allows the creation of dislocation dipoles 'cheaply'. If dipoles are wellseparated, then each dipole gives a positive contribution to the energy which is proportional to the logarithm of the dipole length (the separation distance between the two cores of the dipole). However, for generic configurations of dipoles the sign of the energy contribution is difficult to determine, since it depends strongly upon the relative orientations of the dipoles. In essence, this is a geometric nonlinearity of the system, and most of the effort expended in what follows will be to control the number of dipoles that can form.

From a technical point of view the issue arises as follows: in $\$ 2.2$ we decomposed $D y=\alpha+w$, $\alpha \in[D y]$, since the energy of the displacement $y$ only depends on $\alpha$ due to the periodicity of the potential $\psi$. Consequently, if we have a sequence $u^{n}$ with $\mathcal{E}\left(u^{n}\right)$ uniformly bounded, then this will bound only $\left\|\beta^{n}\right\|_{2}$ for $\beta^{n} \in\left[D u^{n}\right]$, and not $\left\|D u^{n}\right\|_{2}$. In particular, generic minimising sequences cannot be weakly compact.

By exploiting the vertical shift invariance $(\$ 5.2)$ and the horizontal translation invariance $(\$ 5.3)$ of the energy $\mathcal{E}$, we will construct a weakly compact minimising sequence. Having made this special choice of minimising sequence, we use a profile decomposition in $\$ 5.6$. We show that each profile obtained in this way has net Burgers vector zero, leading to the conclusion that the net Burgers vector of the limit remains 1, and proving existence of a minimiser with the properties required. 
5.1. An elementary lower bound. Our eventual goal is to establish a coercivity result for $\mathcal{E}$. We begin with an elementary lower bound that will motivate subsequent constructions.

Let $y=\hat{y}+u, u \in \dot{\mathscr{W}}^{1,2}$, be a trial displacement, $\alpha \in[D y]$, and recall that $\hat{\alpha}=[D \hat{y}]$ is unique. Since $u \in \dot{\mathscr{W}}^{1,2}$, and hence $D u_{b} \rightarrow 0$ as $d_{b} \rightarrow \infty$, it follows that $y$ has a well-defined net Burgers vector in the sense of Definition 2 , and $B[y]=B[\hat{y}]=1$.

Let

$$
\beta:=\alpha-\hat{\alpha}
$$

this 1-form satisfies the property that

$$
\int_{\partial C} \beta \neq 0 \quad \text { if and only if } \quad \int_{\partial C} \alpha \neq \int_{\partial C} \hat{\alpha}
$$

that is, dislocation cores present in $\beta$ are those that are introduced by the addition of $u$ to $\hat{y}$.

Remark 5.1. We note that $\beta_{b}$ does not necessarily belong to $[-1 / 2,1 / 2]$, and hence is not a bond length 1-form, so the definitions of $\$ 2.5$ do not strictly apply; however, it remains a 1-form in the sense of [4, §3.1]. As $\int_{\partial C} \beta \in\{0,1,-1\}$ for all $C \in \mathcal{C}$, we shall therefore slightly abuse our notation and refer to dislocation cores in $\beta$ as the cells $C \in \mathcal{C}$ for which

$$
\int_{\partial C} \beta= \pm 1
$$

We also define $\mathcal{C}^{+}[\beta], \mathcal{C}^{-}[\beta]$ and $\mathcal{C}^{ \pm}[\beta]$ in the obvious way.

Next, we define $z: \mathcal{B} \rightarrow \mathbb{Z}$ via

$$
D u=\beta+z,
$$

which is compactly supported since $\beta, D u \in \ell^{2}(\mathcal{B})$. We shall see in $\$ 5.2$ that the support of $z$ can be thought of as a union of branch cuts connecting dislocation dipoles.

With this notation, we obtain the following result.

Lemma 5.2. For any $u \in \dot{\mathscr{W}}^{1,2}$ with $D u=\beta+z$ as in (5.2) and for any $\epsilon>0$, we have

$$
\mathcal{E}(u) \geq\left(\frac{1}{2} \psi^{\prime \prime}(0)-\epsilon\right)\|\beta\|_{\ell^{2}}^{2}-\sum_{b \in \mathcal{B}} \psi^{\prime}\left(\hat{\alpha}_{b}\right) z_{b}+\langle\delta \mathcal{E}(0), u\rangle-C_{\epsilon},
$$

where $C_{\epsilon}>0$ is a constant that is independent of $u$.

Proof. This estimate arises from the expression 4.12); using the periodicity of the potential $\psi$, we can write

$$
\mathcal{E}(u)=\sum_{b \in \mathcal{B}}\left(\psi\left(\hat{\alpha}_{b}+\beta_{b}\right)-\psi\left(\hat{\alpha}_{b}\right)-\psi^{\prime}\left(\hat{\alpha}_{b}\right) \beta_{b}\right)-\sum_{b \in \mathcal{B}} \psi^{\prime}\left(\hat{\alpha}_{b}\right) z_{b}+\langle\delta \mathcal{E}(0), u\rangle .
$$

Define the function

$$
g(s, t):=\left\{\begin{aligned}
\frac{\psi(t+s)-\psi(t)-\psi^{\prime}(t) s}{s^{2}} & s \neq 0, \\
\frac{1}{2} \psi^{\prime \prime}(t) & s=0 .
\end{aligned}\right.
$$

By assumption $(\psi 5)$ in $\$ 3.1, g(s, 0) \geq \frac{1}{2} \psi^{\prime \prime}(0)$ for any $|s| \leq 1 / 2$.

Since $g$ is uniformly continuous on $[-1 / 2,1 / 2] \times[-\tau, \tau]$ for some $\tau>0$, it follows that for each $\epsilon>0$ there exists $\delta(\epsilon)>0$ such that

$$
g(s, t) \geq \frac{1}{2} \psi^{\prime \prime}(0)-\epsilon \quad \text { for } \quad|s| \leq \frac{1}{2}+\delta(\epsilon) \text { and }|t| \leq \delta(\epsilon) .
$$

Next, we note that 4.9 implies

$$
\left|\hat{\alpha}_{b}\right| \leq \frac{1}{2 \pi d_{b}} \quad \text { and } \quad\left|\beta_{b}\right|=\left|\alpha_{b}-\hat{\alpha}_{b}\right| \leq \frac{1}{2}+\frac{1}{2 \pi d_{b}} .
$$

Hence there exists $R_{0}>0$ such that, for $d_{b} \geq R_{0}$,

$$
g\left(\beta_{b}, \hat{\alpha}_{b}\right) \geq \frac{1}{2} \psi^{\prime \prime}(0)-\epsilon,
$$


which can equivalently be stated as

$$
\psi\left(\hat{\alpha}_{b}+\beta_{b}\right)-\psi\left(\hat{\alpha}_{b}\right)-\psi^{\prime}\left(\hat{\alpha}_{b}\right) \beta_{b} \geq\left(\frac{1}{2} \psi^{\prime \prime}(0)-\epsilon\right)\left|\beta_{b}\right|^{2} \quad \text { for } d_{b} \geq R_{0} .
$$

It may be checked that

$$
\#\left\{b \mid d_{b}<R_{0}\right\} \lesssim R_{0}^{2}
$$

and since $\psi, \psi^{\prime}$ and $\beta$ are uniformly bounded, it therefore follows that

$$
\sum_{b \in \mathcal{B}}\left(\psi\left(\hat{\alpha}_{b}+\beta_{b}\right)-\psi\left(\hat{\alpha}_{b}\right)-\psi^{\prime}\left(\hat{\alpha}_{b}\right) \beta_{b}\right) \geq\left(\frac{1}{2} \psi^{\prime \prime}(0)-\epsilon\right) \sum_{b \in \mathcal{B}}\left|\beta_{b}\right|^{2}-C R_{0}^{2} .
$$

We can think of $\|\beta\|_{2}^{2}$ as estimating elastic stored energy. In the following sections we will establish several results on $z=D u-\beta$, which will eventually allow us to bound the remaining terms $\langle\delta \mathcal{E}(0), u\rangle$ and $\sum_{b} \psi^{\prime}\left(\hat{\alpha}_{b}\right) z_{b}$ in (5.3).

5.2. Dipoles \& Branchcuts. Let $y=\hat{y}+u, u \in \dot{\mathscr{W}}^{1,2}$, be a trial displacement, $\alpha \in[D y]$, and let $\beta, z$ be defined by (5.2). While $\alpha$ and hence $\beta$ are uniquely defined (except in borderline cases when $\alpha_{b} \in\{ \pm 1 / 2\}$ ), one can exploit the vertical shift invariance of the lattice (encoded in assumption $(\psi 1)$, periodicity of $\psi$ ) to construct equivalent displacements $\tilde{u} \in \dot{\mathscr{W}}^{1,2}$,

$$
\tilde{u}:=u+U
$$

where $U: \Lambda \rightarrow \mathbb{Z}$ and $U \in \mathscr{W}_{0}$, and hence modify the $z$ component.

If we let $\tilde{y}:=\hat{y}+\tilde{u}$, then clearly, $\alpha \in[D \tilde{y}]$ and this leads to the same definition of $\beta$. Crucially, though, $D \tilde{u}-\beta \neq D u-\beta$. We can therefore ask how to choose $U$ in an "optimal" way. It turns out that minimizing the total length of the branch cuts is a useful choice, which amounts to minimizing $\|D u+D U-\beta\|_{1}=\|z+D U\|_{1}$. Since $z$ has compact support, a minimizer clearly exists, but it need not be unique; see Figure 5. We may therefore assume, without loss of generality, that $u$ satisfies the discrete minimal connection property (DMCP)

$$
\|D u-\beta\|_{1}=\|z\|_{1}=\min _{Z: \Lambda \rightarrow \mathbb{Z}}\|D u+D Z-\beta\|_{1} .
$$

This minimality condition is similar to the idea of minimal connections, introduced in [5].

We will now establish various properties of the structure of $z$ defined in (5.2). In particular, we will show that $z$ can be decomposed into a sum $\sum z^{m}$ and that the support of each $z^{m}$ is analogous to a branch cut for a dipole.

Lemma 5.3. Let $u \in \dot{\mathscr{W}}^{1,2}$ satisfy the DMCP (5.6) and suppose Du= $\beta+z$ as in (5.2). Then we can write

$$
z=\sum_{m=1}^{M} z^{m},
$$

where $M=\# \mathcal{C}^{+}[\beta]$ is the number of dipoles contained in $\beta$ and $z^{m}: \mathcal{B} \rightarrow\{-1,0,+1\}$, $m=1, \ldots, M$, satisfy the following properties:

(1) $z_{b_{i}}^{m}=1$ on a sequence of bonds $\left(b_{i}\right)_{i=0}^{n}$ such that

(a) $\partial b_{i}$ and $\partial b_{i+1}$ share a common 0 -cell for each $i=0, \ldots, n-1$,

(b) $b_{0} \in \partial C_{m}^{-}$and $-b_{n} \in \partial C_{m}^{+}$where $C_{m}^{+} \in \mathcal{C}^{+}[\beta]$ and $C_{m}^{-} \in \mathcal{C}^{-}[\beta]$.

(2) $z_{b}^{m}=0$ for bonds outside the set $\left\{ \pm b_{i} \mid i=0, \ldots n\right\}$.

Proof. The result is geometrically intuitive; see Figure 5. We therefore postpone a complete proof to Appendix A.1. 


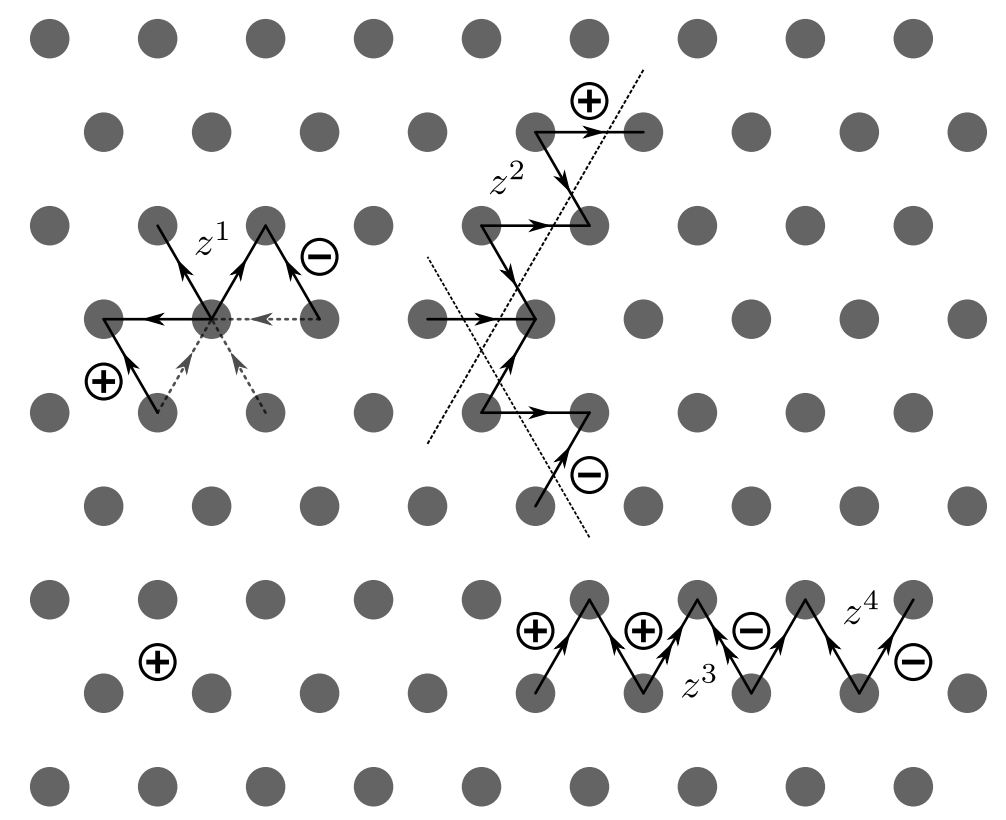

FIGURE 5. A typical example of the support of a minimal $z$ and its decomposition into $z^{m}$ for a given distribution of dipoles. An arrow means that $z_{b}=1$ on the bond pointing in the direction of the arrow, and a double arrow means $z_{b}=2$. The grey dashed bonds on the left of the diagram show an alternative definition of $z$ with the same minimal norm, and the black dashed lines show two lines which show that $z^{2}$ can be decomposed into 2 straight cuts.

We will say that $z^{m}$ connects the dislocation cores $C_{m}^{+}$and $C_{m}^{-}$. Moreover, we obtain the following corollary.

Corollary 5.4. Each 1-form $z^{m}$ in the decomposition (5.7) can be identified with a shortest path in the dual lattice between $C_{m}^{+} \in \mathcal{C}^{+}[\alpha]$ and $C_{m}^{-} \in \mathcal{C}^{-}[\alpha]$, and further

$$
\left\|z^{m}\right\|_{1}=\operatorname{hop}_{2}\left(C_{m}^{+}, C_{m}^{-}\right) .
$$

Proof. First, we observe that, due to the DMCP (5.6) and the decomposition proven in Lemma 5.3 ,

$$
\|z\|_{1}=\sum_{m=1}^{M}\left\|z^{m}\right\|_{1} .
$$

That is, if $z_{b}^{m}, z_{b}^{m^{\prime}} \neq 0$, then $z_{b}^{m}, z_{b}^{m^{\prime}}$ have the same sign.

The construction employed in the proof of Lemma 5.3 identifies a sequence of bonds $b_{i}$ and cells $C_{m, i}$ such that $b_{i},-b_{i+1} \in \partial C_{m, i}, z_{b_{i}}^{m}=1$. Using the natural identification of cells with points in the dual lattice, this implies that $C_{m, i}$ are adjacent in the dual lattice, and furthermore that $b_{i}$ can be identified with edges connecting these cells; this leads to the fact that

$$
\left\|z^{m}\right\|_{1} \geq \operatorname{hop}_{2}\left(C_{m}^{+}, C_{m}^{-}\right) .
$$

To prove the converse, we take the path in the dual lattice corresponding to $z^{m}$, adjoin a shortest path between $C_{m}^{+}$and $C_{m}^{-}$in the dual lattice, and thus obtain a closed dual lattice path. We construct a polygonal closed path in $\mathbb{R}^{2}$ by connecting the barycentres of the cells along the path and define $U$ to be the characteristic function of the bounded interior of this loop in $\mathbb{R}^{2}$.

It is now straightforward to check that by defining $\tilde{z}^{m}:=z^{m}+D U$ and $\tilde{z}:=z-z^{m}+\tilde{z}^{m}$

$$
\|\tilde{z}\|_{1} \leq \sum_{m^{\prime}=1}^{M}\left\|z^{m^{\prime}}\right\|_{1}-\left\|z^{m}\right\|_{1}+\left\|\tilde{z}^{m}\right\|_{1} .
$$


Since $U$ is a compactly supported integer shift as in (5.5), the discrete minimal connection property (5.6) implies that $\left\|z^{m}\right\|_{1} \leq\left\|\tilde{z}^{m}\right\|_{1}=\operatorname{hop}_{2}\left(C_{m}^{+}, \overline{C_{m}^{-}}\right)$, completing the proof.

Later on it will be convenient to assume that each cut $z^{m}$ is made up of at most two straight cuts: straight cuts are defined to be 1 -forms $z: \mathcal{B} \rightarrow\{-1,0,1\}$ for which there exists a line $L:=\left\{x^{C}+t a_{i} \mid t \in \mathbb{R}\right\}$, where $x^{C}$ is the barycentre of some $C \in \mathcal{C}$ and $a_{i}$ a nearest neighbour direction, such that $z_{b} \neq 0$ if and only if the bond satisfies $b \cap L \neq \emptyset$,

$$
\operatorname{clos}\left\{x \in \mathbb{R}^{2} \mid x \in b \in \mathcal{B}, z_{b} \neq 0\right\}
$$

is a connected set, and $z_{b}>0$ either exclusively on bonds in the directions $a_{i+1}$ and $a_{i+2}$ or in the directions $a_{i-1}$ and $a_{i-2}$. We will say that a straight cut 'lies in the direction $a_{j}$ ' whenever $a_{i}= \pm a_{j}$ in the definition of the corresponding $L$. See the cut depicted in the centre of Figure 5 for a visualisation of the definition.

In the next lemma, we show that we can always choose the decomposition (5.7) such that each $z^{m}$ is composed of at most 2 straight cuts. We will refer to any $u$ as in the conclusion of Lemma 5.5 as satisfying the straight cuts property.

Lemma 5.5. Let $u \in \dot{\mathscr{W}}^{1,2}$, and $D u=\beta+z$ as in $(5.2)$. Then there exists $\tilde{u} \in \dot{\mathscr{W}}^{1,2}$ satisfying the DMCP (5.6) as well as D $\tilde{u}=\beta+\sum_{m=1}^{\# \mathcal{C}^{+}[\beta]} z^{m}$ where each $z^{m}$ is the sum of at most 2 straight cuts.

Proof. The idea is to show that we may always find a shortest path in the dual lattice between any pair of cells which is made up of 2 straight segments. It is intuitively clear from Figure 5 that this can always be done.

A complete proof is postponed until Appendix A.2.

5.3. Shifting the Origin. Suppose that $y=\hat{y}+u$, where $u \in \dot{\mathscr{W}}^{1,2}$ satisfies the DMCP (5.6). For any $C \in \mathcal{C}$, we define the affine transformation

$$
F^{C} \xi:=\left\{\begin{aligned}
\xi+x^{C} & \text { if the triangle } C \text { points upwards, } \\
\mathrm{R}_{6}\left(\xi+x x^{C}\right) & \text { if the triangle } C \text { points downwards, }
\end{aligned}\right.
$$

where $\mathrm{R}_{6}$ denotes the rotation through angle $\pi / 3$. Thus, $F^{C}$ maps the lattice onto the lattice, and $C_{0}$ onto the cell $C$.

Next, let

$$
u^{C}:=u \circ F^{C}+\hat{y} \circ F^{C}-\hat{y} .
$$

It follows that $\hat{y}(\xi)+u^{C}(\xi)=\hat{y}\left(F^{C} \xi\right)+u\left(F^{C} \xi\right)$ for all lattice points $\xi \in \Lambda$, so that there are corresponding bond length 1-forms $\alpha^{C} \in\left[D \hat{y}+D u^{C}\right]$ satisfying

$$
\alpha^{C}=\alpha \circ F^{C} \text {. }
$$

As before, define $\beta^{C}:=\alpha^{C}-\hat{\alpha}$. According to these definitions,

$$
\hat{y}\left(F^{C} \xi\right)-\hat{y}(\xi):=\frac{1}{2 \pi}\left(\arg \left(\xi+x^{C}\right)-\arg (\xi)\right) ;
$$

if we make this function single-valued by introducing a compact polygonal branch cut passing through the barycentres of a shortest dual lattice path between $C_{0}$ and $C$, then it is a straightforward exercise to show that

$$
\left|D\left(\hat{y} \circ F^{C}\right)_{b}-D \hat{y}_{b}\right| \lesssim d_{b}^{-2}
$$

therefore $u^{C} \in \dot{\mathscr{W}}^{1,2}$, and

$$
\begin{aligned}
\mathcal{E}\left(u^{C}\right) & =E\left(\hat{y}+u^{C} ; \hat{y}\right) \\
& =E\left(\hat{y} \circ F^{C}+u \circ F^{C} ; \hat{y}\right) \\
& =E\left(\hat{y} \circ F^{C}+u \circ F^{C} ; \hat{y} \circ F^{C}\right)+E\left(\hat{y} \circ F^{C} ; \hat{y}\right) \\
& =\mathcal{E}(u)
\end{aligned}
$$


noting that the the first term on the third line is simply a resummation of $\mathcal{E}(u)$, and the second term vanishes.

For each $C \in \mathcal{C}^{+}[\alpha]$, we can replace $u^{C}$ with $\tilde{u}^{C}=u^{C}+U$ for some $U: \Lambda \rightarrow \mathbb{Z}$, such that $\left\|D u^{C}+D U-\beta^{C}\right\|_{1}$ is minimal, i.e. $\tilde{u}^{C}$ satisfies the DMCP (5.6). We obtain that

$$
\mathcal{E}\left(\tilde{u}^{C}\right)=\mathcal{E}\left(u^{C}\right)=\mathcal{E}(u) .
$$

To summarize, we have constructed a corrector displacement $\tilde{u}^{C} \in \dot{\mathscr{W}}^{1,2}$ with the same energy as $u$, but for which $C \in \mathcal{C}^{+}[\alpha]$ has been shifted to the origin. Upon minimising $\left\|D \tilde{u}^{C}-\beta^{C}\right\|_{1}$ amongst all choices $C \in \mathcal{C}^{+}[\alpha]$, we obtain the following result.

Lemma 5.6. Let $v \in \dot{\mathscr{W}}^{1,2}$, then there exists $u \in \dot{\mathscr{W}}^{1,2}$ such that $\mathcal{E}(u)=\mathcal{E}(v)$ and such that the discrete optimal connection property holds:

(DOCP) There exists $\alpha \in[D(\hat{y}+u)]$ such that

$$
\|D u-\beta\|_{1}=\min _{C \in \mathcal{C}^{+}[\alpha]} \min _{U: \Lambda \rightarrow \mathbb{Z}}\left\|D u^{C}+D U-\beta^{C}\right\|_{1},
$$

where $\beta=\alpha-\hat{\alpha}, u^{C}$ is defined by (5.10) and $\beta^{C}=\alpha \circ F^{C}-\hat{\alpha}$, where $F^{C}$ is defined in (5.9).

The crucial property that we obtain from the DOCP (5.11) is a bound on the distance between the necessary core at $C_{0}$ and all negative cores.

Lemma 5.7. Suppose $u \in \dot{\mathscr{W}}^{1,2}$ satisfies the DOCP (5.11) and let $z=\sum_{m=1}^{M} z^{m}$ according to (5.7). Then,

$$
\operatorname{hop}_{2}\left(C_{0}, C_{m}^{-}\right) \geq \operatorname{hop}_{2}\left(C_{m}^{+}, C_{m}^{-}\right), \quad \text { for } m=1, \ldots, M,
$$

where we recall that $z^{m}$ connects the cores $C_{m}^{+} \in \mathcal{C}^{+}[\alpha]$ and $C_{m}^{+} \in \mathcal{C}^{-}[\alpha]$.

Proof. Suppose the converse for contradiction. Then there exists $m$ and a dual lattice path connecting $C_{0}$ to $C_{m}^{-}$which is strictly shorter than $\operatorname{hop}_{2}\left(C_{m}^{+}, C_{m}^{-}\right)$. Letting $F:=F^{C_{m}^{+}}$and $v:=u^{C_{m}^{+}}$,

$$
D v-\beta \circ F=z \circ F+\tilde{z}=\sum_{m} z^{m} \circ F+\tilde{z}
$$

where $\tilde{z}$ is the contribution coming from the branch cut in $\hat{y} \circ F-\hat{y}$. Consider the closed curve passing from $F C_{m}^{+}=C_{0}$ to $F C_{0}$ along the branch cut, then along a shortest lattice path between $F C_{0}$ and $F C_{m}^{-}$, and then back to $F C_{m}^{+}$along the support of $z^{m} \circ F$. This is a closed curve, and by a similar argument to that in Corollary 5.4. we can define $w \in \mathscr{W}_{0}$ as $w(\xi)=1$ for $\xi \in \Lambda$ inside the curve, and 0 outside. It can then be checked that $v+w$ has a corresponding $\bar{z}$ which satisfies

$$
\|\bar{z}\|_{1}=\|z \circ F\|-\left\|z^{m} \circ F\right\|+\operatorname{hop}_{2}\left(C_{0}, C_{m}^{-}\right)=\sum_{i \neq m}\left\|z^{i}\right\|_{1}+\operatorname{hop}_{2}\left(C_{0}, C_{m}^{-}\right)<\|z\|_{1},
$$

the required contradiction.

As a corollary we obtain the following stronger property.

Corollary 5.8. Suppose $u \in \dot{\mathscr{W}}^{1,2}$ satisfies the DOCP (5.11) and let $z=\sum_{m=1}^{M} z^{m}$ according to (5.7). Then, for any $m \in\{1, \ldots, M\}$ and for any cell $C \in \mathcal{C}$ such that $z_{b}^{m} \neq 0$ for some $b \in \partial C$,

$$
\operatorname{hop}_{2}\left(C_{0}, C\right) \geq \operatorname{hop}_{2}\left(C_{m}^{+}, C\right) .
$$

Proof. Lemma 5.7 states that, if $C^{-} \in \mathcal{C}^{-}$and $C^{+} \in \mathcal{C}^{+}$are connected by $z^{m}$, then

$$
\operatorname{hop}_{2}\left(C_{0}, C^{-}\right) \geq \operatorname{hop}_{2}\left(C^{+}, C^{-}\right) \text {. }
$$

It is clear that any subpath of a shortest path in a graph is also a shortest path. By the construction of $z^{m}, C$ lies on a shortest path between $C^{+}$and $C^{-}$, and therefore

$$
\operatorname{hop}_{2}\left(C^{+}, C^{-}\right)=\operatorname{hop}_{2}\left(C^{+}, C\right)+\operatorname{hop}_{2}\left(C, C^{-}\right) .
$$


The triangle inequality for paths 2.3 now directly implies

$$
\operatorname{hop}_{2}\left(C_{0}, C\right) \geq \operatorname{hop}_{2}\left(C^{+}, C\right) .
$$

5.4. Estimating $\langle\delta \mathcal{E}(0), u\rangle$. In $\$ 5.2$ and $\$ 5.3$, we showed that for any $u \in \dot{\mathscr{W}}^{1,2}$, we can find $\tilde{u} \in \dot{\mathscr{W}}^{1,2}$ such that $\mathcal{E}(u)=\mathcal{E}(\tilde{u})$, and for which the corresponding branch cuts $z$ satisfy the $\mathbf{D O C P}(5.11)$. We are now in a position to exploit the chosen structure of $z$ to derive compactness for minimising sequences.

Our first step is to provide a stronger bound on $\delta \mathcal{E}(0)$. We have already shown that $|\langle\delta \mathcal{E}(0), u\rangle| \lesssim\|D u\|_{\ell^{2}}$, but this will not be sufficient since our estimates so far only provide a bound on $\beta$, and not on $D u$ itself. Therefore we need to estimate $|\langle\delta \mathcal{E}(0), u\rangle|$ only in terms of $\|\beta\|_{2}$.

Lemma 5.9. For each $u \in \dot{\mathscr{W}}^{1,2}$, let $\beta_{u}:=\beta$ be defined through (5.1).

There exists a constant $C>0$ such that

$$
\langle\delta \mathcal{E}(0), u\rangle \leq C\left\|\beta_{u}\right\|_{2} \quad \forall u \in \dot{\mathscr{W}}^{1,2} \quad \text { satisfying the DOCP } 5.11 \text {. }
$$

We provide the proof of this fundamental estimate throughout the remainder of this section.

Recall the definition of $\xi_{0}$ from $\$ 2.2$. For any $\xi \in \Lambda$, it is always possible to express the difference $\xi-\xi_{0}$ as

$$
\xi-\xi_{0}=n a_{i}+m a_{i+1},
$$

for some nearest neighbour lattice direction $a_{i}$ and some $n, m \in \mathbb{N} \cup\{0\}$ with $n \neq 0$ unless $\xi=\xi_{0}$. We then define the path $\Gamma_{\xi}$ to be

$$
\Gamma_{\xi}:=\sum_{j=0}^{n-1}\left(\xi_{0}+j a_{i}, \xi_{0}+(j+1) a_{i}\right)+\sum_{j=0}^{m-1}\left(\xi_{0}+n a_{i}+j a_{i+1}, \xi_{0}+n a_{i}+(j+1) a_{i+1}\right) ;
$$

cf. Figure 6. Integrating $D u_{b}$ along $\Gamma_{\xi}$, we obtain

$$
\begin{aligned}
|u(\xi)| & =\left|\int_{\Gamma_{\xi}} D u\right|=\left|\int_{\Gamma_{\xi}} \beta+z\right| \\
& \leq\left|\Gamma_{\xi}\right|^{1 / 2}\left(\sum_{b \in \Gamma_{\xi}}\left|\beta_{b}\right|^{2}\right)^{1 / 2}+\left|\int_{\Gamma_{\xi}} z\right| \\
& \lesssim|\xi|^{1 / 2}\|\beta\|_{2}+\left|\int_{\Gamma_{\xi}} z\right|
\end{aligned}
$$

using the Cauchy-Schwarz inequality. We now bound the final term in (5.14).

Lemma 5.10. Suppose $u \in \dot{\mathscr{W}}^{1,2}$ satisfies the DOCP 5.11) and the straight cuts property (cf. Lemma 5.5); then

$$
\left|\int_{\Gamma_{\xi}} z\right| \lesssim \min \left\{|\xi|^{2}, \# \mathcal{C}^{+}[\alpha]\right\}
$$

Proof. First, we note that for any straight cut $z^{\prime}$,

$$
\left|\int_{\Gamma_{\xi}} z^{\prime}\right| \leq 1
$$

This follows from the fact that all $b \in \mathcal{B}$ for which $z_{b}^{\prime}=1$ can be written as

$$
\left(\xi+n a_{j}, \xi+n a_{j}+a_{j+1}\right) \text { or }\left(\xi+n a_{j}, \xi+n a_{j}+a_{j+2}\right)
$$




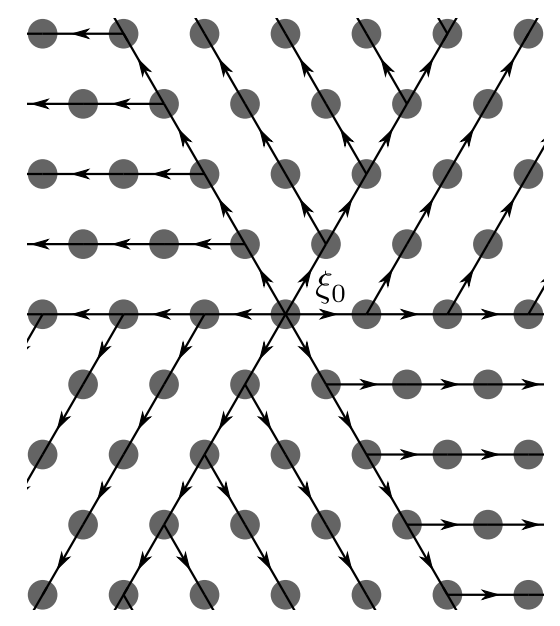

Figure 6. An illustration of the definition of $\Gamma_{\xi}$.

for some $\xi \in \Lambda, n \in \mathbb{N}$ and some nearest neighbour lattice direction $a_{j}$, and the definition of $\Gamma_{\xi}$. Using the straight cuts property, we find that

$$
\left|\int_{\Gamma_{\xi}} z\right| \leq \sum_{m}\left|\int_{\Gamma_{\xi}} z^{m}\right| \leq 2 \# \mathcal{C}^{+}[\alpha]
$$

To prove the second bound, enumerate the bonds $b_{j} \in \Gamma_{\xi}$, beginning at with the bond $b_{1}=\left(\xi_{0}, \xi_{0}+a_{i}\right)$. Each $b_{j} \in \partial C^{b_{j}}$ for some $C^{b_{j}} \in \mathcal{C}$. Since $u$ satisfies the DOCP, Corollary 5.8 , $z_{b_{j}}^{m} \neq 0$ implies that if $z^{m}$ connects to $C^{+} \in \mathcal{C}^{+}[\alpha]$, then

$$
\operatorname{hop}_{2}\left(C^{+}, C^{b_{j}}\right) \leq \operatorname{hop}_{2}\left(C_{0}, C^{b_{j}}\right) \leq \operatorname{hop}_{2}\left(C_{0}, C^{b_{0}}\right)+2 j
$$

where we have repeatedly applied the triangle inequality (2.3). Further application of the triangle inequality implies that the sequence of sets

$$
\left\{C \in \mathcal{C} \mid \operatorname{hop}_{2}\left(C, C^{b_{j}}\right) \leq \operatorname{hop}_{2}\left(C_{0}, C^{b_{0}}\right)+2 j\right\}
$$

for $j=1, \ldots,\left|\Gamma_{\xi}\right|$ is increasing, and it is easy to see that

$$
\#\left\{C \in \mathcal{C} \mid \operatorname{hop}_{2}\left(C, C^{b_{j}}\right) \leq \operatorname{hop}_{2}\left(C_{0}, C^{b_{0}}\right)+2 j\right\} \lesssim j^{2} .
$$

Since by 2.4 each cell can contain at most one dislocation core, we must therefore have

$$
\left|\int_{\Gamma_{\xi}} z\right| \lesssim\left|\Gamma_{\xi}\right|^{2} \lesssim|\xi|^{2} .
$$

Next, we show that the number of cores can be bounded in terms of $\beta$. This is intuitive, since, as discussed in [28], each core stores a positive amount of elastic energy. Let $C \in \mathcal{C}^{ \pm}[\beta]$, then Jensen's inequality implies

$$
\int_{\partial C}|\beta|^{2} \geq \frac{1}{3}\left|\int_{\partial C} \beta\right|^{2}=\frac{1}{3}
$$

Hence, we obtain

$$
\# \mathcal{C}^{ \pm}[\beta] \leq 3\|\beta\|_{2}^{2}
$$

Proof of Lemma 5.9. We now combine Lemma 5.10, Lemma 4.4 and 5.16 to estimate

$$
\begin{aligned}
|\langle\delta \mathcal{E}(0), u\rangle| & \leq \sum_{\xi \in \Lambda}|f(\xi)||u(\xi)| \\
& \lesssim \sum_{\xi \in \Lambda}\left\{\|\beta\|_{2}|\xi|^{-5 / 2}+\min \left(\|\beta\|_{2}^{2},|\xi|^{2}\right)|\xi|^{-3}\right\} .
\end{aligned}
$$


We note that the $|\xi|^{-5 / 2} \in \ell^{1}(\Lambda)$, so that the first term is bounded above by $C\|\beta\|_{2}$. The second term splits into

$$
\sum_{\xi \in \Lambda} \min \left(\|\beta\|_{2}^{2},|\xi|^{2}\right)|\xi|^{-3} \leq \sum_{|\xi| \leq\|\beta\|_{2}}|\xi|^{-1}+\|\beta\|_{2}^{2} \sum_{|\xi|>\|\beta\|_{2}}|\xi|^{-3}
$$

Straightforward radial estimates yield the bounds

$$
\sum_{|\xi| \leq\|\beta\|_{2}}|\xi|^{-1} \lesssim\|\beta\|_{2} \quad \text { and } \quad \sum_{|\xi|>\|\beta\|_{2}}|\xi|^{-3} \lesssim\|\beta\|_{2}^{-1}
$$

Combining the previous estimates, we obtain the stated result.

5.5. Estimating $\sum_{b \in \mathcal{B}} z_{b} \psi^{\prime}\left(\hat{\alpha}_{b}\right)$. In this section, we estimate the second group in (5.3). Unlike in our previous estimates, which are generic, we now resort to precise quantitative bounds based heavily on our assumption $(\psi 5)$.

Lemma 5.11. Suppose that $u \in \dot{\mathscr{W}}^{1,2}$ satisfies the DOCP (5.11), then for any cut $z^{m}$ connecting to a dipole $C^{+} \in \mathcal{C}^{+}[\alpha]$ to $C^{-} \in \mathcal{C}^{-}[\alpha]$, we have the lower bound:

$$
\sum_{b \in \mathcal{B}} z_{b}^{m} \psi^{\prime}\left(\hat{\alpha}_{b}\right) \geq-\psi^{\prime \prime}(0) \frac{\operatorname{arcsinh}(2 / \sqrt{3})}{\pi}-c_{0} \operatorname{hop}_{2}\left(C_{0}, C^{-}\right)^{-1}
$$

where $c_{0}>0$ is independent of $u$.

Remark 5.12. We have no reason to believe that the estimate (5.17) is sharp. It is sufficient for our purpose, due only to the fairly strong technical assumption $(\psi 5)$ made in $\$ 3.1$.

The complete proof of Lemma 5.11 is given in Appendix A, but since this is a crucial part of our analysis, we provide a brief sketch.

Sketch of the proof of Lemma 5.11. A crucial consequence of the DOCPis (5.13), since this inequality says that the dipoles described by each $z^{m}$ satisfy

$$
\begin{aligned}
& \text { either: } \operatorname{hop}_{2}\left(C_{0}, C^{+}\right) \leq \operatorname{hop}_{2}\left(C_{0}, C^{-}\right) ; \\
& \text {or: } \quad \operatorname{hop}_{2}\left(C_{0}, C^{-}\right)<\operatorname{hop}_{2}\left(C_{0}, C^{+}\right), \quad \text { but } \operatorname{hop}_{2}\left(C^{+}, C^{-}\right) \leq \operatorname{hop}_{2}\left(C_{0}, C^{-}\right) .
\end{aligned}
$$

Since there is a positive dislocation core present in $C_{0}$, dipoles in the first category give a positive contribution to the sum, since the repulsive force between $C^{+}$and $C_{0}$ dominates.

In the second case, the attractive forces between $C_{0}$ and $C^{-}$dominate, hence these dipoles give a negative contribution to the energy. Requiring the straight cuts property derived in Lemma 5.5 allows us to obtain estimates on the terms in the sums

$$
\sum_{b \in \mathcal{B}} \psi^{\prime}\left(\hat{\alpha}_{b}\right) z_{b}^{m}
$$

using (4.9), and estimating sums in terms of integrals that can be evaluated explicitly. Thus, we obtain explicit bounds for various different cases in terms of hop ${ }_{2}\left(C_{0}, C^{-}\right)$and $\operatorname{hop}_{2}\left(C^{+}, C^{-}\right)$. These estimates are logarithmic, and hence turn out to essentially depend upon the ratio

$$
\frac{\operatorname{hop}_{2}\left(C^{+}, C^{-}\right)}{\operatorname{hop}_{2}\left(C_{0}, C^{-}\right)} \leq 1
$$

the final result is the bound stated in (5.17).

We have now collected all estimates required to obtain a coercivity result. Although we state the result for general $u \in \dot{\mathscr{W}}^{1,2}$, we will only require it later for $u$ satisfying the DOCP.

Theorem 5.13. Let $u \in \dot{\mathscr{W}}^{1,2}$, then there exists $\alpha \in[D \hat{y}+D u]$ and $\beta=\alpha-\hat{\alpha}$, such that

$$
\mathcal{E}(u) \geq c_{1}\|\beta\|_{2}^{2}+c_{2} \# \mathcal{C}^{ \pm}[\beta]-c_{3},
$$

where $c_{i}>0$ are independent of $u$. 
Remark 5.14. We note that since we will show $\|\beta\|_{2}^{2} \gtrsim \# \mathcal{C}^{ \pm}[\beta]$, we could write (5.18) more concisely as

$$
\mathcal{E}(u) \geq c_{1}\|\beta\|_{2}^{2}-c_{3} .
$$

Proof. The bound is clearly invariant under the vertical shift and horizontal shift and rotation transformations we applied in $\$ 5.2$ and $\$ 5.3$. Without loss of generality, we may therefore assume that $u$ satisfies the DOCP (5.11). Moreover, according to Remark 2.1 we can choose $\alpha \in[D \hat{y}+D u]$ in such a way that $\alpha_{b}=0$ on any bond that lies on the intersection between two cores.

Summing (5.17) over $m$, we find that

$$
\sum_{b \in \mathcal{B}} z_{b} \psi^{\prime}\left(\hat{\alpha}_{b}\right) \geq-\psi^{\prime \prime}(0) \frac{\operatorname{arcsinh}(2 / \sqrt{3})}{2 \pi} \# \mathcal{C}^{ \pm}[\beta]-c_{0} \sum_{C \in \mathcal{C}^{-}[\beta]} \operatorname{hop}_{2}\left(C, C_{0}\right)^{-1} \text {. }
$$

for some $c_{0}>0$.

The second group in (5.19) can be estimated using the fact that each cell can contain no more that 1 dislocation core, so that for any $\delta>0$, there is a constant $C_{\delta}$ such that

$$
\sum_{C \in \mathcal{C}^{-}[\beta]} \operatorname{hop}_{2}\left(C, C_{0}\right)^{-1} \geq-\delta \# \mathcal{C}^{ \pm}[\beta]-C_{\delta} .
$$

Bringing together (5.3), (5.9) and $(5.19)$, we have that for arbitrary $\epsilon>0$ and $\delta>0$

$$
\mathcal{E}(u) \geq\left(\frac{1}{2} \psi^{\prime \prime}(0)-\epsilon\right)\|\beta\|_{2}^{2}-\left(\psi^{\prime \prime}(0) \frac{\operatorname{arcsinh}(2 / \sqrt{3})}{2 \pi}+\delta\right) \# \mathcal{C}^{ \pm}[\alpha]-C_{\epsilon, \delta}
$$

Since we assumed that $\alpha_{b}=0$ on any bond that is adjacent to two cores (cf. Remark 2.1), we obtain from $(5.15)$ that

Since

$$
\|\beta\|_{2}^{2} \geq \frac{1}{3} \# \mathcal{C}^{ \pm}[\beta]
$$

$$
\frac{1}{3}>\frac{\operatorname{arcsinh}(2 / \sqrt{3})}{\pi} \approx 0.314
$$

the result follows by taking $\epsilon$ and $\delta$ small enough.

5.6. Existence of Minimisers of $\mathcal{E}$. With the coercivity result of Theorem 5.13 in place, we are now in a position to apply the Direct Method and establish existence of a minimiser of $\mathcal{E}$ in $\dot{\mathscr{W}}^{1,2}$.

Take a sequence $u^{n} \in \dot{\mathscr{W}}^{1,2}$ such that

$$
\mathcal{E}\left(u^{n}\right) \rightarrow \inf _{u \in \dot{\mathscr{W}} 1,2} \mathcal{E}(u) .
$$

Referring back to 5.3 , we may assume that $u^{n}$ satisfies the DOCP. Let $\alpha^{n} \in\left[D \hat{y}+D u^{n}\right]$ satisfy the condition of Theorem 5.13, and $\beta^{n}:=\alpha^{n}-\hat{\alpha}$. Theorem 5.13 then implies that $\beta^{n}$ has a weakly convergent subsequence in $\ell^{2}(\mathcal{B})$. In the next lemma, we also obtain convergence of $D u^{n}$.

Lemma 5.15. Suppose $u^{n} \in \dot{\mathscr{W}}^{1,2}$ is a minimising sequence for which each $u^{n}$ satisfies the DOCP (5.11). Then there exists a subsequence which converges weakly in $\dot{\mathscr{W}}^{1,2}$, and the corresponding $z^{n}$ converges weakly in any $\ell^{p}(\mathcal{B})$ with $1<p<2$.

Proof. Corollary 5.13 implies that, selecting a subsequence of $u^{n}$ (not relabelled), we may assume that $\beta^{n} \rightarrow \beta$ weakly in $\ell^{2}(\mathcal{B})$ and that $M:=\# \mathcal{C}^{+}\left[\beta^{n}\right]$ is constant along the sequence.

Let $D u^{n}=\beta^{n}+z^{n}$. Lemma 5.3 implies that each $z^{n}$ can be decomposed into

$$
z^{n}=\sum_{m=1}^{M} z^{n, m}
$$


Let $B$ be any finite sum of positively-oriented cells. Since weak convergence in $\ell^{2}(\mathcal{B})$ implies pointwise convergence, it follows that

$$
\int_{\partial B} \beta^{n}=-\int_{\partial B} z^{n} \rightarrow N \in \mathbb{Z} \quad \text { as } n \rightarrow \infty .
$$

We enumerate the cores $C^{n, m} \in \mathcal{C}^{ \pm}\left[\alpha^{n}\right], n \in \mathbb{N}, m=1, \ldots, 2 M+1$. Let $\mathcal{M}_{\text {bdd }}$ be the set of indices of cores that remain at a bounded distance from the origin, that is,

$$
\mathcal{M}_{\text {bdd }}:=\left\{m \in\{1, \ldots, 2 M+1\} \mid \sup _{n \in \mathbb{N}} d_{C^{n, m}}<+\infty\right\} .
$$

Since the core centres $x^{C^{n, m}}$ with $m \in \mathcal{M}_{\text {bdd }}$ can only take a finite number of positions, we can extract a further subsequence (not relabelled) so that they are constant.

We therefore observe that

$$
A:=\sum \mathcal{C}^{ \pm}[\beta]=\sum_{m \in \mathcal{M}_{\mathrm{bdd}}} C^{n, m}, \quad \text { for all } n .
$$

Then, for any finite sum of cells $B, B \supset A$, we have

$$
\lim _{n \rightarrow \infty} \int_{\partial B} \beta^{n}=\lim _{n \rightarrow \infty} \int_{\partial A} \beta^{n}=\int_{\partial A} \beta=: N \in \mathbb{Z} .
$$

We aim to show that $N=0$. For all $n$ sufficiently large, applying Jensen's inequality implies

$$
\int_{\partial A}\left|\beta^{n}\right|^{2} \geq \frac{1}{|\partial A|}\left|\int_{\partial A} \beta^{n}\right|^{2}=\frac{N}{|\partial A|} .
$$

Let $B_{0}^{0} \supset A$ be a finite sum of cells that form a convex lattice polygon, and let

$$
B_{k}^{0}=B_{k-1}^{0} \cup\left\{C: \overline{\partial C} \cap B_{k-1}^{0} \neq \emptyset\right\},
$$

for $k \in \mathbb{N}$. By considering all possible corners for a convex lattice polygon it is straightforward to show that $\left|\partial B_{k}^{0}\right|=\left|\partial B_{k-1}^{0}\right|+6$. Since $\lim _{n \rightarrow \infty} \int_{\partial B_{k}^{0}} \beta^{n}=N \in \mathbb{Z}$, there exist $n_{k}$ such that $\int_{\partial B_{i}^{0}} \beta^{n_{k}}=N$ for $i=1, \ldots, k$, and hence,

$$
\left\|\beta^{n_{k}}\right\|_{2}^{2} \geq \sum_{i=0}^{k} \int_{\partial B_{i}^{0}}\left|\beta^{n_{k}}\right|^{2} \geq \sum_{i=0}^{k} \frac{N}{\left|\partial B_{k}^{0}\right|} \gtrsim N \log (k) .
$$

Since $\left\|\beta^{n_{k}}\right\|_{2}$ is bounded, we obtain that $N=0$. We can therefore conclude that, for any finite sum of positively oriented cells $b, B \supset A$,

$$
\int_{\partial B} z^{n} \rightarrow 0 \quad \text { as } \quad n \rightarrow \infty
$$

Using a concentration compactness argument, we now show that we can "group" those cores which diverge into sums of cells with net Burgers vector zero.

To that end, define the lattice translation operators $F_{m}^{n}:=F_{m}^{C_{m}^{n}}$ as in $(5.9)$, where $C_{m}^{n} \in$ $\mathcal{C}^{+}\left[\beta^{n}\right]$ and $\beta_{m}^{n}:=\beta^{n} \circ F_{m}^{n}$. Note that

$$
\left\|\beta_{m}^{n}\right\|_{2}=\left\|\beta^{n}\right\|_{2}
$$

so $\beta_{m}^{n}$ is a bounded sequence for each $m$, and we can select a subsequence such that $\beta_{m}^{n} \rightarrow \beta_{m}$ for some $\beta_{m}$ in $\ell^{2}(\mathcal{B})$ and for each $m=1, \ldots, M$. As above, it follows that there exists a finite sum of positively-oriented cells $A^{m}$ which contains $C_{0}$ and all dislocation cores of $\beta_{m}$, and is such that for any sum of positively-oriented cells $B$ containing $A^{m}$ as a subsum,

$$
-\int_{\partial B} z^{n} \circ F_{m}^{n}=\int_{\partial B} \beta_{m}^{n}=0 \text { for } n \text { sufficiently large. }
$$


Let $A_{m}^{n}:=\left(F_{m}^{n}\right)^{-1} A^{m}$, then we have shown that, for all $n$ sufficiently large, all dislocation cores in $\beta^{n}$ lie within the set

$$
S^{n}:=\bigcup_{m=1}^{M} A_{m}^{n} .
$$

We are now ready to establish that $\left\|z^{n}\right\|_{1}$ is bounded. Since each $z^{n}$ satisfies the DMCP, $\left\|z^{n}\right\|_{\infty} \leq M$. We therefore simply need to rule out the possibility that $\# \operatorname{supp}\left\{z^{n}\right\} \rightarrow \infty$ as $n \rightarrow \infty$ (i.e., that the branch cut lengths diverge).

Fix some $m \in\{1, \ldots, M\}$ and suppose that the core $C_{m}^{n} \in \mathcal{C}^{+}\left[\beta^{n}\right]$ is connected to $K_{1}^{n} \in$ $\mathcal{C}^{-}\left[\beta^{n}\right]$ via a "cut" $z^{n, m}$. We claim that $K_{1}^{n} \in A^{n, m}$.

If this were false and $K_{1}^{n} \in A_{\ell_{1}}^{n}$ where $\ell_{1} \neq m$, then there must be $L_{1}^{n} \in \mathcal{C}^{+}\left[\beta^{n}\right], L_{1}^{n} \in A_{\ell_{1}}^{n}$, which is connected to another core $K_{2}^{n} \in \mathcal{C}^{-}\left[\beta^{n}\right]$ outside of $A_{\ell_{1}}^{n}$ and outside $A_{m}^{n}$. Upon iterating this construction, we find a series of cores $K_{1}^{n}, L_{1}^{n}, K_{2}^{n}, L_{2}^{n}, \ldots$, which must eventually repeat.

Let $L_{1}^{n}:=C_{m}^{n}$. We know, by construction of the groups $A_{\ell}^{n}$ that $\operatorname{hop}_{2}\left(L_{i}^{n}, K_{i+1}^{n}\right) \rightarrow \infty$ but $\operatorname{hop}_{2}\left(K_{i}^{n}, L_{i}^{n}\right)$ is bounded as $n \rightarrow \infty$ for each $i$. This clearly contradicts the DMCP and hence the DOCP.

Hence, the claim that $K_{1}^{n} \in A^{n, m}$ follows, and this immediately implies that $\left\|z^{n}\right\|_{1}$ is bounded.

Since $\left\|z_{n}\right\|_{1}$ is bounded and $\ell^{1}$ compactly embeds into $\ell^{p}$ for any $p>1$, it follows that we may extract a further subsequence which weakly converges in some $\ell^{p}(\mathcal{B})$ with $1<p<2$. This further imples that both $z^{n}$ and $\beta^{n}$ converge weakly in $\ell^{2}(\mathcal{B})$, and in particular that $u^{n}$ converges weakly in $\dot{\mathscr{W}}^{1,2}$, as required.

We now complete the proof of our main result.

Proof of Theorem 4.5. Invoking Lemma 5.15, suppose that the minimising sequence $u^{n} \rightarrow u$ in $\dot{\mathscr{W}}^{1,2}$, which is a candidate minimiser for $\mathcal{E}$, and furthermore that the corresponding $z^{n}$ converges weakly in $\ell^{p}(\mathcal{B})$ with $1<p<2$. It remains to show that $\lim \inf \mathcal{E}\left(u^{n}\right) \geq \mathcal{E}(u)$. Recall from 4.12 that

$$
\mathcal{E}(u)=\sum_{b \in \mathcal{B}}\left[\psi\left(\hat{\alpha}_{b}+D u_{b}\right)-\psi\left(\hat{\alpha}_{b}\right)-\psi^{\prime}\left(\hat{\alpha}_{b}\right) D u_{b}\right]+\langle\delta \mathcal{E}(0), u\rangle
$$

Since the second term is a bounded linear functional, it is weakly continuous. Using (4.9), it may be shown that the linear functional $L$ defined to be

$$
L(z):=\sum_{b \in \mathcal{B}} \psi^{\prime}\left(\hat{\alpha}_{b}\right) z_{b}
$$

is in $\left(\ell^{p}(\mathcal{B})\right)^{*}$ for all $p<2$, and is therefore also weakly continuous along the sequence. From (5.4) we obtain that there exists $R_{0}, \lambda>0$ such that, for $d_{b} \geq R_{0}$,

$$
\psi\left(\hat{\alpha}_{b}+D u_{b}^{n}\right)-\psi\left(\hat{\alpha}_{b}\right)-\psi^{\prime}\left(\hat{\alpha}_{b}\right) \beta_{b}^{n} \geq \lambda\left|\beta_{b}^{n}\right|^{2} \geq 0
$$

We can therefore apply Fatou's lemma to obtain

$$
\liminf _{n \rightarrow \infty} \sum_{b \in \mathcal{B}} \psi\left(\hat{\alpha}_{b}+D u_{b}^{n}\right)-\psi\left(\hat{\alpha}_{b}\right)-\psi^{\prime}(\hat{\alpha}) \beta_{b}^{n} \geq \sum_{b \in \mathcal{B}} \psi\left(\hat{\alpha}_{b}+D u_{b}\right)-\psi\left(\hat{\alpha}_{b}\right)-\psi^{\prime}\left(\hat{\alpha}_{b}\right) \beta_{b},
$$

and thus, in combination with the weak continuity of $L$ and $\delta \mathcal{E}(0)$ along the minimising sequence,

$$
\inf _{v \in \dot{\mathscr{W}}^{1,2}} \mathcal{E}(v)=\liminf _{n \rightarrow \infty} \mathcal{E}\left(u^{n}\right) \geq \mathcal{E}(u) \geq \inf _{v \in \dot{\mathscr{W}}^{1,2}} \mathcal{E}(v),
$$

completing the proof of Theorem 4.5 . 


\section{Conclusion}

We have presented a model which has allowed us to analyse the stability of screw dislocations under anti-plane deformation, and we have obtained the surprising result that single screw dislocations exist as globally stable states, i.e. they are global energy minimisers among all finite energy displacements. Further, we then showed that configurations with arbitrarily many dislocations of arbitrary sign are locally stable, as long as the dislocation cores are suitably separated, but such configurations do not appear to be globally stable.

Our work suggests two immediate directions for future study. Firstly, our analysis relies crucially on the technical assumption $(\psi 5)$ made in $\$ 3.1$. It would be of interest to understand the extent to which this assumption could be weakened, but to do so would require a more qualitative estimate than the quantitative one made in Lemma 5.11, and would require a deeper insight into the geometry of dipole interaction. Secondly, it would be interesting to understand which other lattice defects are globally stable states; this seems far from clear to us at present.

\section{ACKNOWLEDGEMENTS}

The authors would like to thank Florian Theil for originally posing the problem of describing the existence of dislocations in atomistic problems, Adriana Garroni for discussions regarding the literature on dislocations, and Sylvia Serfaty for highlighting the work contained in [5] to us.

\section{Appendix A. Analysis of Branchcuts: Proofs}

In this appendix, we detail the proofs of various geometrical lemmas from $\$ 5.2$.

A.1. Proof of Lemma 5.3. We will prove this lemma algorithmically. First, if $z_{b}=0$ everywhere, then the result is trivial. Next, we note that $\beta$ contains an even number of dislocation cores, and $\# \mathcal{C}^{+}[\beta]=\# \mathcal{C}^{-}[\beta]<+\infty$ since $u \in \dot{\mathscr{W}}^{1,2}$. We therefore enumerate $C_{i}^{+} \in \mathcal{C}^{+}[\beta]$.

Put $C_{1,0}=C_{1}^{+}$. Since $C_{1}^{+}$is a positive dislocation core and $z_{b}$ is integer-valued, it follows that at least one bond $b_{1} \in \partial C_{1}^{+}$satisfies $z_{b}>0$. Let $C_{1,1}$ be the cell such that $-b_{1} \in C_{1,1}$. There are now 2 possibilities: either $C_{1,1} \in \mathcal{C}^{-}[\beta]$, in which case we stop, or we can find another bond $b_{2} \in \partial C_{1,1}$ such that $z_{b}>0$. Iterating, we obtain a (possibly infinite) sequence of cells $C_{1, j}$ and bonds $b_{j}$.

We now claim that no two bonds $b_{j}=b_{k}$ with $j \neq k$ in this sequence, and consequently the sequence terminates; suppose the converse for contradiction. Let $j$ and $k$ be indices such that $j<k$ and $k-j$ is minimal over all pairs of indices such that $b_{j}=b_{k}$. Define a polygonal curve $P$ passing through the barycentres of the cells $x^{C_{1, j}}, \ldots, x^{C_{1, k}}$. $P$ is a simple continuous closed curve, since the cells $C_{1, j}, \ldots, C_{1, k-1}$ are distinct by definition, and $C_{1, j}=C_{1, k}$. Hence, $P$ partitions $\mathbb{R}^{2}$ into a bounded set $\Omega$ (the interior of $P$ ) and an unbounded set, $\mathbb{R}^{2} \backslash \Omega$. Define $\tilde{u}=u \mp \mathbb{1}_{\Omega}$, taking the sign according to whether $P$ traverses $\partial \Omega$ in an anticlockwise or clockwise direction respectively.

It is now straightforward to check that $D \tilde{u}=D u$ except on the bonds $b_{i}$. For each of the bonds $b_{i}$,

$$
D \tilde{u}_{b_{i}}=D u_{b_{i}}-1=\beta_{b_{i}}+z_{b_{i}}-1 \text {, }
$$

but since $z_{b_{i}} \geq 1$, this contradicts the DMCP, (5.6). It follows that the sequence $b_{i}$ contains no two identical bonds, and as $z_{b}$ has compact support, the sequence must terminate at a negative dislocation core.

Define $z_{b}^{1}= \pm 1$ if $\pm b \in\left\{b_{1}, \ldots, b_{n}\right\}$, and $z_{b}^{1}=0$ otherwise, and then consider iterating the procedure described above starting at $C_{i}^{+}$, but using the criterion at each step that each bond in the sequence must satisfy

$$
z_{b}-\sum_{m=1}^{i-1} z_{b}^{m}>0 .
$$


This leads to a sequence 1 -forms, $z^{i}$; the resulting 1 -form

$$
z_{b}-\sum_{i=1}^{N} z_{b}^{i}
$$

must be identically zero. If not, then the same technique as used above shows that that either the $\mathbf{D M C P}$ is violated, or else $\operatorname{supp}\{z\}$ is infinite, and hence $u \notin \dot{\mathscr{W}}^{1,2}$.

To complete the proof of Lemma 5.3, we simply need to show that for 2 adjacent bonds in the support of any $z^{m}, \partial b_{i} \cap \partial b_{i+1}$ is a single 0 -cell. This is clear, since by definition $b_{i},-b_{i+1} \in \partial C_{m, i}$, $b_{i} \neq b_{i+1}$ and $\left|\partial C_{m, i}\right|=3$.

A.2. Proof of Lemma 5.5. We now show that we can always choose $z^{m}$ to be made up of 2 straight cuts. Recall that Corollary 5.4 states that $\left\|z^{m}\right\|_{1}=\operatorname{hop}_{2}\left(C_{m}^{+}, C_{m}^{-}\right)$.

Next, define the 2-cell hop operators $H_{i}$ for $i=1, \ldots 6$ which act on 2-cells by taking a cell $C$ to the first cell 'in the direction $a_{i}$ ', that is, the positively-oriented cell which satisfies $C^{\prime} \neq C$,

$$
x^{C}+\frac{\sqrt{3}}{2} a_{i} \in C^{\prime} .
$$

It is straightforward to check this is well-defined; see Figure 7. We can represent paths in the dual lattice by words taken from the alphabet of operators $\left\{H_{1}, \ldots, H_{6}\right\}$. In general however, the representation is non-unique - to see this, it is clear that for $C_{2}$ in Figure $7, H_{2} C_{2}=H_{1} C_{2}$. As with the vectors $a_{i}$, we define

$$
H_{i+6 m}:=H_{i}
$$

for any $m \in \mathbb{Z}$.

We note the following properties of the operators $H_{i}$, which may be easily checked:

(1) The orbit of the group of operators generated by $\left\{H_{1}^{2}, \ldots, H_{6}^{2}\right\}$ acting on any cell $C$ is a lattice.

(2) The operators $H_{i}^{2}$ and $H_{j}$ commute for any $i$ and $j$.

(3) For any cell $C$ and any $i$, one of the following is true: $H_{i} C=H_{i+1} C$ or $H_{i} C=H_{i-1} C$.

(4) If $H_{i} C=H_{j} C$, then $H_{i} H_{k}^{2 m} C=H_{j} H_{k}^{2 m} C$, and if $H_{i} C \neq H_{j} C$, then $H_{i} H_{k}^{2 m} C \neq$ $H_{j} H_{k}^{2 m} C$.

(5) $H_{i+3} H_{i}=H_{i+3} H_{i}$ is the identity map for any $i$.

(6) $H_{i} C \neq H_{i \pm 2} C$ for any cell $C$ and for any $i$.

Setting $N:=\left\|z^{m}\right\|_{1}$, we claim that for any pair of cells $C_{m}^{ \pm}$, it is possible to write shortest paths in the dual lattice as a word of the form

$$
H_{i+1}^{N-k} H_{i}^{k}
$$

for some $i$ and some $k \in\{1, \ldots, N\}$. By the definition of the hopping operators, it is clear that such words represent a sequence of cells lying on the lines $x^{C_{m}^{-}}+t a_{i}$ and $x^{C_{m}^{+}}-t a_{i+1}$.

We now prove the claim: first, we show that any shortest path must be able to be written as a word made up of only 2 of the operators $H_{i}$ and $H_{i+1}$. Suppose this is false, for contradiction. (6) implies that we may assume that it contains a segment which may be written as

$$
H_{i+2} H_{i}^{m} H_{i-2} \quad \text { or } \quad H_{i-2} H_{i}^{m} H_{i+2}
$$

for some $i$ and some $m \in \mathbb{N}$. Since both cases are similar, we consider only the first. If $C$ is the first cell in this subsequence, $\mathrm{H}_{i-2} \mathrm{C} \neq \mathrm{H}_{i-3} \mathrm{C}$, or else $\mathrm{H}_{i} \mathrm{H}_{i-2} \mathrm{C}=\mathrm{H}_{i} \mathrm{H}_{i-3} \mathrm{C}=\mathrm{C}$ by (5), and there exists a shorter path. (3) therefore implies

$$
H_{i-2} C=H_{i-1} C .
$$

Invoking property (3) again, either

$$
H_{i} H_{i-2} C=H_{i} H_{i-1} C=H_{i-1}^{2} C \quad \text { or } \quad H_{i} H_{i-2} C=H_{i+1} H_{i-2} C=C .
$$

Once more, the second case results in a contradiction; hence repeatedly using (2),

$$
H_{i+2} H_{i}^{2 k+1} H_{i-2} C=H_{i}^{2 k} H_{i+2} H_{i} H_{i-2} C=H_{i}^{2 k} H_{i+2} H_{i-1}^{2} C=H_{i}^{2 k} H_{i-1} C,
$$




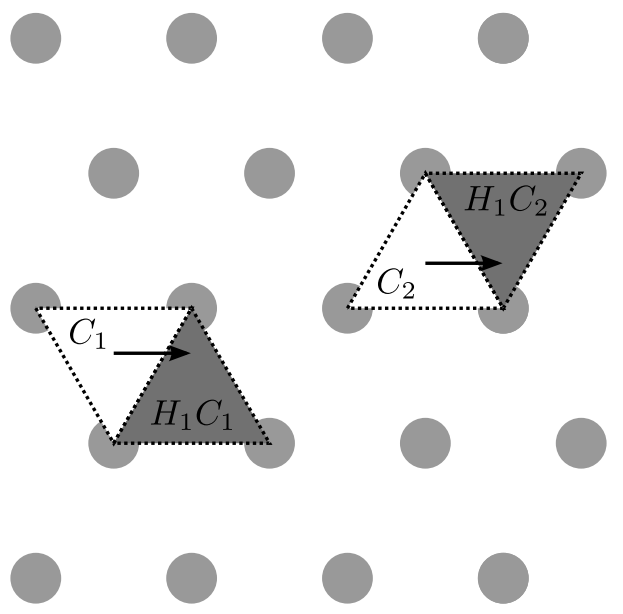

FiguRE 7. This illustration shows the operation of the operator $H_{a_{1}}$ on two representative cells, $C_{1}$ and $C_{2}$.

implying a contradiction in the case where $m$ is odd. In the case that $m$ is even, repeatedly using (2), (A.2) and (5), we have

$$
H_{i+2} H_{i}^{2 k} H_{i-2} C=H_{i}^{2 k} H_{i+2} H_{i-2} C=H_{i}^{2 k} H_{i+2} H_{i-1} C=H_{i}^{2 k} C,
$$

obtaining another contradiction; it follows that it is impossible that every shortest path can be written as a word containing no more than 2 of the operators $H_{i}$.

We next show that if the two operators are $H_{i}$ and $H_{i+2}$ for some $i$, we may rewrite the word in terms of $H_{i}$ and $H_{i-1}$, or $H_{i+1}$ and $H_{i}$. In (A.3), we showed that for any cell $C$ contained in a shortest path $H_{i} H_{i-2} C=H_{i-1}^{2} C$. Suppose that a shortest path is represented as a product of $H_{i-2}$ and $H_{i}$. Then each pair $H_{i} H_{i-2}$ may be replaced by $H_{i-1}^{2}$, and using (2) to permute each of these pairs to the right, we eventually obtain one of

$$
H_{i}^{m} H_{i-1}^{2 n} \quad \text { or } \quad H_{i-2}^{m} H_{i} H_{i-1}^{2 n} .
$$

In the first case, the proof is complete. In the second case, it must be that $H_{i} H_{i-1}^{2 n}=H_{i-1}^{2 n+1}$ or else $m=0$, since otherwise (3) implies

$$
H_{i-2}^{m} H_{i} H_{i-1}^{2 n}=H_{i-2}^{m} H_{i+1} H_{i-1}^{2 n}=H_{i-2}^{m-1} H_{i-1}^{2 n},
$$

which is a contradiction. Hence we have proved the claim, and in fact since we have obtained a shortest path in the form A.1, the lemma is proven for this particular case.

Finally, we consider a general word made up of only the operators $H_{i}$ and $H_{i+1}$. Now consider a word of the form $H_{i+1} H_{i}^{m} H_{i+1}$. If $m$ is even, then we can generate a new word corresponding to a shortest path $H_{i+1}^{2} H_{i}^{m}$. If $m$ is odd, then we can write a new shortest path as

$$
H_{i+1} H_{i} H_{i+1} H_{i}^{m-1} .
$$

But then using (3), this must be able to be written either as

$$
H_{i+1}^{3} H_{i}^{m-1} \text {, or } H_{i+2} H_{i-1} H_{i+2} H_{i}^{m-1},
$$

where the second case results in a contradiction. It is now possible to check that this implies the full conclusion, since by these arguments we can always transform a general word composed of $H_{i}$ and $H_{i+1}$ in one of the form A.1.

A.3. Proof of Lemma 5.11. To prove this lemma, we use Lemma 5.5 to assert that $z$ should be made up of straight cuts. This will allow us to make estimates for each straight segment, which depend upon on the orientation of each segment. We divide the lattice into 'sextants' by defining

$$
\mathcal{S}^{i}:=\left\{x \in \mathbb{R}^{2} \mid \exists \lambda>0, \mu \geq 0 \text { such that } x=\lambda a_{i}+\mu a_{i+1}\right\} .
$$


and rings $\mathcal{R}^{i}$ by defining $\mathcal{R}^{-1}=\emptyset, \mathcal{R}^{0}:=C_{0}$, and then

$$
\mathcal{R}^{i}=\operatorname{clos} \bigcup\left\{C \in \mathcal{C} \backslash\left(\mathcal{R}^{i-1} \cup \mathcal{R}^{i-2}\right) \mid \operatorname{clos}(C) \cap \operatorname{clos}\left(\mathcal{R}^{i-1}\right) \neq \emptyset\right\} .
$$

We will say a straight cut $z$ is:

(1) tangential if $\operatorname{supp}\{z\} \subseteq \mathcal{S}^{i}$ and the cut direction is $a_{i+2}$, or equivalently $\operatorname{supp}\{z\} \subseteq$ $\mathcal{S}^{i} \cap \mathcal{R}^{r}$ for some $r$, and

(2) radial if $\operatorname{supp}\{z\} \subseteq \mathcal{S}^{i}$ and it has direction $a_{i}$ or $a_{i+1}$.

It may be checked that for any straight cut, there exist $C^{+}, C^{-} \in \mathcal{C}$ such that

$$
\int_{\partial C^{ \pm}} z= \pm 1
$$

We separate the full result into 2 further lemmas, which give precise estimates for each of these classes of straight cuts, before combining them to complete the proof for the general case. By Taylor expanding $\psi^{\prime}\left(\hat{\alpha}_{b}\right)$ around 0 we write

$$
\sum_{b \in \mathcal{B}} \psi^{\prime}\left(\hat{\alpha}_{b}\right) z_{b}=\sum_{b \in \mathcal{B}} \psi^{\prime \prime}(0) \hat{\alpha}_{b} z_{b}+\frac{1}{6} \psi^{(4)}\left(s_{b}\right)\left(\hat{\alpha}_{b}\right)^{3} z_{b} .
$$

We proceed to estimate the first terms in the summand, by estimating on the radial and tangential straight segments of $z^{m}$ separately.

Lemma A.1. For a tangential cut $z^{\tan }$ with $\left\|z^{\tan }\right\|_{1}=\operatorname{hop}_{2}\left(C^{+}, C^{-}\right)=l$ on ring $\mathcal{R}^{r}$, we have the following estimate:

$$
\sum_{b \in \mathcal{B}} z_{b}^{\tan } \hat{\alpha}_{b} \geq-\frac{1}{2 \pi} \arctan \left(\frac{2\lfloor\min (l, 2 r-l) / 2\rfloor+1}{(r-2 / 3) \sqrt{3}}\right)-O\left(r^{-1}\right) .
$$

Proof. First, we appeal to symmetry. If $b=\left(\xi, \xi+a_{j}\right) \in \mathcal{S}_{i}$, then applying the reflection

$$
R=\frac{1}{3}\left(a_{i}+a_{i+1}\right) \otimes\left(a_{i}+a_{i+1}\right)-a_{i+2} \otimes a_{i+2},
$$

it is straightforward to check that if $b^{\prime}=(R \xi, R \zeta)$, then

$$
\hat{\alpha}_{b}=-\hat{\alpha}_{b^{\prime}} .
$$

This means that if a tangential cut crosses the line of symmetry $\left\{t\left(a_{i}+a_{i+1}\right) \mid t \in \mathbb{R}\right\}$, then some of the bond contributions cancel. It follows that we need only consider the case where all bonds in the support on $z^{\text {tan }}$ lie on one side of this line of symmetry, since this is the worst case. For such cuts, it may be checked that $l \leq r$.

Identifying $z^{\tan }$ with a dual lattice path, we may enumerate $b_{k} \in \operatorname{supp}\left\{z^{\tan }\right\}$ 'along the path', and elementary geometry now shows that $\hat{\alpha}_{b_{k}}$ has alternating sign as $k$ increases. Letting $\xi^{r}:=\frac{1}{2}\left(r-\frac{2}{3}\right)\left(a_{i}+a_{i+1}\right)$ if $i$ is odd, $\xi^{r}:=\frac{1}{2}\left(r-\frac{1}{3}\right)\left(a_{i}+a_{i+1}\right)$ if $i$ is even, it is straightforward to show that each bond $b_{k}$ can be represented as one of

$$
\left(\xi^{r}+s a_{i+2}, \xi^{r}+s a_{i+2}+a_{i}\right) \quad \text { or } \quad\left(\xi^{r}+s a_{i+2}, \xi^{r}+s a_{i+2}+a_{i+1}\right)
$$

where $s \in\left\{0, \frac{1}{2}, \ldots, \frac{r-1}{2}\right\}$. Elementary trigonometry now allows us to calculate that

$$
\begin{aligned}
2 \pi \hat{\alpha}_{b} z_{b}^{\tan } & = \pm\left[\arctan \left(\frac{s+1 / 2}{\left|\xi^{r+1}\right|}\right)-\arctan \left(\frac{s}{\left|\xi^{r}\right|}\right)\right] \\
\text { or } & \pm\left[\arctan \left(\frac{s-1 / 2}{\left|\xi^{r+1}\right|}\right)-\arctan \left(\frac{s}{\left|\xi^{r}\right|}\right)\right]
\end{aligned}
$$


respectively for the cases above. We therefore have that

$$
\begin{aligned}
2 \pi \sum_{b} z_{b}^{\tan } \hat{\alpha}_{b} & =\sum_{t=1}^{\lfloor l / 2\rfloor} \arctan \left(\frac{t+s_{0}+1 / 2}{\left|\xi^{r+1}\right|}\right)-2 \arctan \left(\frac{t+s_{0}}{\left|\xi^{r}\right|}\right)+\arctan \left(\frac{t+s_{0}-1 / 2}{\left|\xi^{r+1}\right|}\right)+O\left(r^{-1}\right), \\
\text { or } & =\sum_{t=1}^{\lfloor l / 2\rfloor} \arctan \left(\frac{t+s_{0}+1 / 2}{\left|\xi^{r}\right|}\right)-2 \arctan \left(\frac{t+s_{0}}{\left|\xi^{r+1}\right|}\right)+\arctan \left(\frac{t+s_{0}-1 / 2}{\left|\xi^{r}\right|}\right)+O\left(r^{-1}\right),
\end{aligned}
$$

where $s_{0} \in\left\{0, \frac{1}{2}, \ldots, \frac{r-1}{2}\right\}$, and the $O\left(r^{-1}\right)$ term arises from the contribution of at most 2 bonds we have neglected, whose contribution we estimate using (4.9). In the second case, the fact that - arctan is convex for positive arguments implies that the sum is bounded below by 0 . In the first case, for all $t$ in the range of summation,

$$
\frac{t+s_{0}+1 / 2}{\left|\xi^{r+1}\right|} \geq \frac{t+s_{0}}{\left|\xi^{r}\right|} \text { and trivially } \frac{t+s_{0}}{\left|\xi^{r+1}\right|} \leq \frac{t+s_{0}}{\left|\xi^{r}\right|}
$$

Since arctan is increasing, we obtain the lower bound

$$
\begin{aligned}
2 \pi \sum_{b} z_{b}^{\tan } \hat{\alpha}_{b} & \geq \sum_{t=1}^{\lfloor l / 2\rfloor}\left\{\arctan \left(\frac{t+s_{0}-1 / 2}{\left|\xi^{r}\right|}\right)-\arctan \left(\frac{t+s_{0}}{\left|\xi^{r}\right|}\right)\right\}+O\left(r^{-1}\right) \\
& \geq-\arctan \left(\frac{\lfloor l / 2\rfloor+1 / 2}{\left|\xi^{r}\right|}\right)+O\left(r^{-1}\right)
\end{aligned}
$$

using the fact that arctan is positive and increasing for positive arguments. Finally, note that in the case where $l>r$, i.e. the tangential cut crosses the line of symmetry, and we obtain the same estimate but with $2 r-l$ in place of $l$ in the formula above, so using the definition of $\xi^{r}$ gives the result.

Lemma A.2. For a radial cut $z^{\mathrm{rad}}$ such that $\left\|z^{\mathrm{rad}}\right\|_{1}=\operatorname{hop}_{2}\left(C^{+}, C^{-}\right)=l$, either $\left|x^{C^{+}}\right|<\left|x^{C^{-}}\right|$ and

$$
\sum_{b \in \mathcal{B}} z_{b}^{\mathrm{rad}} \hat{\alpha}_{b} \geq 0
$$

or else $\left|x^{C^{+}}\right|>\left|x^{C^{-}}\right|$, and if $C^{-} \in \mathcal{R}^{r}$, then

$$
\left.\sum_{b \in \mathcal{B}} z_{b}^{\mathrm{rad}} \hat{\alpha}_{b} \geq-\frac{1}{\pi} \operatorname{arcsinh}\left(\frac{2\lceil l / 2\rceil}{\sqrt{3}(r-2 / 3)}\right)\right)-O\left(r^{-1}\right) .
$$

Proof. First, we enumerate the bonds in $b_{k} \in\left\{b \in \mathcal{B} \mid z_{b}^{\text {rad }}>0\right\}$, beginning with the bond for which $d_{b}$ is smallest, and proceeding outwards along the cut. Elementary geometry shows that the terms $z_{b_{k}}^{\mathrm{rad}} \hat{\alpha}_{b_{k}}$ are all positive in the case where $b_{1}$ is in one of the directions $a_{i+1}, a_{i+2}$ or $a_{i+3}$, which corresponds to having $\left|x^{C^{+}}\right|<\left|x^{C^{-}}\right|$; this immediately provides the first bound.

In the second case 4.9 implies

$$
\hat{\alpha}_{b_{k}} \geq-\frac{1}{2 \pi d_{b_{k}}} .
$$

Without loss of generality, we assume the cut direction is $a_{i}$, the case with direction $a_{i+1}$ being similar. There are now two cases: $b_{1}$ is either in the direction $a_{i-1}$, or $a_{i-2}$. Further elementary geometry allows us to conclude that in the first case, $d_{b_{1}}=|x|$ for some $x \in \mathcal{S}^{i}$, and in the second, $d_{b_{1}}>|x|$ with $x=x^{C^{-}} \in \operatorname{clos}\left(\mathcal{S}^{i}\right)$. In either case, $d_{b_{2}}$ satisfies the same lower bound as $d_{b_{1}}$, and further, we have that

$$
d_{b_{2 n-1}}, d_{b_{2 n}} \geq\left|x+n a_{i}\right| .
$$

Noting that as $x \in \operatorname{clos}\left(\mathcal{S}^{i}\right)$, it follows that $a_{i} \cdot x \geq 0$ and

$$
\frac{1}{\left|x+t a_{i}\right|}=\frac{1}{\sqrt{|x|^{2}+2 t a_{i} \cdot x+t^{2}}} \leq \frac{1}{\sqrt{|x|^{2}+t^{2}}}
$$


which is a decreasing function of $t$, so we estimate

$$
\sum_{k=1}^{l} \hat{\alpha}_{b_{k}} \geq-\frac{1}{\pi} \sum_{i=0}^{\lceil l / 2\rceil} \frac{1}{\left|x+n a_{i}\right|} \geq-\frac{1}{\pi}\left(\frac{1}{|x|}+\int_{0}^{\lceil l / 2\rceil} \frac{1}{\sqrt{|x|^{2}+s^{2}}} \mathrm{~d} s\right) .
$$

Evaluating the integral, and noting further that $|x| \geq \frac{\sqrt{3}}{2}(r-2 / 3)$, we obtain the conclusion.

We now combine the estimates of Lemma A.1 and Lemma A.2 to obtain an estimate for a general cut $z^{m}$ made up of two straight segments. As we showed in Lemma 5.5, each $z^{m}$ is made up of at most 2 straight segments. It may be checked that each of these segments is either purely radial, purely tangential, or changes from tangential to radial part way along its length, with one bond which crosses $\partial \mathcal{S}^{i}$. All possible cuts satisfying the DMCP and made up of 2 straight segments can therefore be decomposed as either

(1) a tangential cut and 2 radial cuts or

(2) a tangential cut, a radial cut and tangential cut,

where any of these segments could possibly have length 0 , and neglecting the extra bonds mentioned above for now. Recalling the result of Corollary 5.8.

$$
\operatorname{hop}_{2}\left(C_{0}, C_{m}^{-}\right) \geq \operatorname{hop}_{2}\left(C_{0}, C_{m}^{+}\right) .
$$

Consider the first case, letting the two radial segments be of length $l_{1}$ and $l_{2}$ respectively, and the tangential segment of length $l-l_{1}-l_{2}$. If $\left|x^{C_{m}^{+}}\right|<\left|x^{C_{m}^{-}}\right|$and the radial cuts are of non-zero length, then the radial segments have the trivial lower bound, by Lemma A.2. In the worst case, where $l-l_{1}-l_{2}=r$, we have the bound

$$
\sum_{b \in \mathcal{B}} z_{b}^{m} \hat{\alpha}_{b} \geq-\frac{1}{2 \pi} \arctan \left(\frac{r+1}{(r-2 / 3) \sqrt{3}}\right)-O\left(r^{-1}\right)=-\frac{1}{12}-O\left(r^{-1}\right) .
$$

Otherwise, $\left|x^{C_{m}^{+}}\right|>\left|x^{C_{m}^{-}}\right|$, so applying Corollary 5.8, we have that for $C^{\prime} \in \mathcal{R}^{r}$,

$$
2 r \geq \operatorname{hop}_{2}\left(C_{0}, C^{\prime}\right) \geq \operatorname{hop}_{2}\left(C_{m}^{+}, C^{\prime}\right)=l_{1}+l_{2} .
$$

Therefore, applying Lemma A.1 and Lemma A.2.

$$
\begin{array}{r}
\sum_{b \in \mathcal{B}} z_{b} \hat{\alpha}_{b} \geq-\frac{1}{2 \pi}\left(\arctan \left(\frac{2\left\lfloor\left(l-l_{1}-l_{2}\right) / 2\right\rfloor+1}{(r-2 / 3) \sqrt{3}}\right)+2 \operatorname{arcsinh}\left(\frac{2\left\lceil l_{1} / 2\right\rceil}{\sqrt{3}(r-2 / 3)}\right)\right. \\
\left.+2 \operatorname{arcsinh}\left(\frac{2\left\lceil l_{2} / 2\right\rceil}{\sqrt{3}\left(r+\left\lfloor l_{1} / 2\right\rfloor-2 / 3\right)}\right)+O\left(r^{-1}\right)\right) .
\end{array}
$$

By ignoring the floor functions, it is possible to check that under the bound (A.5), the function in parentheses is increasing in both $l_{1}$ and $l_{2}$ if $r$ is suitably large; we therefore have that the maximum must occur when $l=l_{1}+l_{2}$. Hence putting $l_{2}=l-l_{1}$, we have

$$
\sum_{b \in \mathcal{B}} z_{b} \hat{\alpha}_{b} \geq-\frac{1}{\pi}\left(\operatorname{arcsinh}\left(\frac{2\left\lceil l_{1} / 2\right\rceil}{\sqrt{3}(r-2 / 3)}\right)+\operatorname{arcsinh}\left(\frac{2\left\lceil\left(l-l_{1}\right) / 2\right\rceil}{\sqrt{3}\left(r+\left\lfloor l_{1} / 2\right\rfloor-2 / 3\right)}\right)+O\left(r^{-1}\right)\right) .
$$

Dropping the floor and ceiling functions, this estimate is convex in $l_{1}$. The worst cases are therefore $l_{1}=0$ or $l_{1}=l$ and $2\lceil l / 2\rceil=2 r+1$, giving the value

$$
\sum_{b \in \mathcal{B}} z_{b} \hat{\alpha}_{b} \geq-\frac{1}{\pi} \operatorname{arcsinh}\left(\frac{2 r+1}{\sqrt{3}(r-2 / 3)}\right)+O\left(r^{-1}\right)=-\frac{\operatorname{arcsinh}(2 / \sqrt{3})}{\pi}+O\left(r^{-1}\right) .
$$

In the case where we have tangential, radial and tangential segments, similar arguments show that, once more, the worst possible bound arises in the case where the cut is purely radial, giving the same lower bound. 
We now return to the lower order terms in A.4. Then by crudely estimating

$$
\left|\sum_{b \in \mathcal{B}} \frac{1}{6} \psi^{(4)}\left(s_{b}\right)\left(\hat{\alpha}_{b}\right)^{3} z_{b}^{m}\right| \lesssim \sum_{d_{b} \geq r} d_{b}^{-3} \lesssim \frac{1}{r}
$$

and noting that the worst case bounds always have occur when hop ${ }_{2}\left(C_{0}, C_{m}^{-}\right) \simeq r$, we have

$$
\sum_{b \in \mathcal{B}} z_{b}^{m} \psi^{\prime}\left(\hat{\alpha}_{b}\right) \geq-\psi^{\prime \prime}(0) \frac{\operatorname{arcsinh}(2 / \sqrt{3})}{\pi}-c_{0} \operatorname{hop}_{2}\left(C_{0}, C_{m}^{-}\right)^{-1} \text {. }
$$

\section{REFERENCES}

[1] R. Alicandro and M. Cicalese. Variational analysis of the asymptotics of the $X Y$ model. Arch. Ration. Mech. Anal., 192(3):501-536, 2009.

[2] Roberto Alicandro, Marco Cicalese, and Marcello Ponsiglione. Variational equivalence between GinzburgLandau, XY spin systems and screw dislocations energies. Indiana Univ. Math. J., 60(1):171-208, 2011.

[3] R. J. Amodeo and N. M. Ghoniem. Dislocation dynamics. i. a proposed methodology for deformation micromechanics. Phys. Rev. B, 41:6958-6967, 1990.

[4] M. P. Ariza and M. Ortiz. Discrete crystal elasticity and discrete dislocations in crystals. Arch. Ration. Mech. Anal., 178(2), 2005.

[5] Haïm Brezis, Jean-Michel Coron, and Elliott H. Lieb. Harmonic maps with defects. Comm. Math. Phys., $107(4), 1986$.

[6] V. V. Bulatov and W. Cai. Computer Simulations of Dislocations, volume 3 of Oxford Series on Materials Modelling. Oxford University Press, 2006.

[7] Paolo Cermelli and Giovanni Leoni. Renormalized energy and forces on dislocations. SIAM J. Math. Anal., 37(4):1131-1160 (electronic), 2005.

[8] Sergio Conti, Adriana Garroni, and Stefan Müller. Singular kernels, multiscale decomposition of microstructure, and dislocation models. Arch. Ration. Mech. Anal., 199(3):779-819, 2011.

[9] Reinhard Diestel. Graph theory, volume 173 of Graduate Texts in Mathematics. Springer, Heidelberg, fourth edition, 2010.

[10] V. Ehrlacher, C. Ortner, and A. Shapeev. in preparation.

[11] Helmut Föll. Defects in crystals, March 2013. http://www.tf.uni-kiel.de/matwis/amat/def_en/.

[12] A. Garroni and S. Müller. Г-limit of a phase-field model of dislocations. SIAM J. Math. Anal., 36(6):19431964 (electronic), 2005.

[13] Adriana Garroni, Giovanni Leoni, and Marcello Ponsiglione. Gradient theory for plasticity via homogenization of discrete dislocations. J. Eur. Math. Soc. (JEMS), 12(5):1231-1266, 2010.

[14] Adriana Garroni and Stefan Müller. A variational model for dislocations in the line tension limit. Arch. Ration. Mech. Anal., 181(3):535-578, 2006.

[15] John Price Hirth and Jens Lothe. Theory of Dislocations. Krieger Publishing Company, Malabar, Florida, 1982.

[16] T. Hudson, J. Mason, and C. Ortner. in preparation.

[17] T. Hudson and C. Ortner. Manuscript.

[18] Derek Hull and David J Bacon. Introduction to dislocations, volume 37. Butterworth-Heinemann, 2011.

[19] M. Koslowski, A. M. Cuitiño, and M. Ortiz. A phase-field theory of dislocation dynamics, strain hardening and hysteresis in ductile single crystals. J. Mech. Phys. Solids, 50(12):2597-2635, 2002.

[20] M. Luskin, C. Ortner, and B. Van Koten. Formulation and optimization of the energy-based blended quasicontinuum method. Comput. Methods Appl. Mech. Engrg., 253, 2013.

[21] M.A. Peletier M.G.D. Geers, R.H.J. Peerlings and L. Scardia. Asymptotic behaviour of a pile-up of infinite walls of edge dislocations. ArXiv e-prints, May 2012.

[22] R. Miller and E. Tadmor. A unified framework and performance benchmark of fourteen multiscale atomistic/continuum coupling methods. Modelling Simul. Mater. Sci. Eng., 17, 2009.

[23] E. Orowan. Zur kristallplastizität. iii. Zeitschrift für Physik, 89:634-659, 1934.

$[24]$ C. Ortner and A. Shapeev. Interpolants of lattice functions for the analysis of atomistic/continuum multiscale methods. ArXiv e-prints, 1204.3705, 2012.

[25] C. Ortner and A. V. Shapeev. Analysis of an Energy-based Atomistic/Continuum Coupling Approximation of a Vacancy in the 2D Triangular Lattice. Math. Comp., forthcoming.

[26] C. Ortner and F. Theil. Nonlinear elasticity from atomistic mechanics, 2012. arXiv:1202.3858v3.

[27] M. Polanyi. Über eine art gitterstörung, die einen kristall plastisch machen könnte. Zeitschrift für Physik, 89:660-664, 1934.

[28] Marcello Ponsiglione. Elastic energy stored in a crystal induced by screw dislocations: from discrete to continuous. SIAM J. Math. Anal., 39(2), 2007. 
[29] D Rodney, Y Le Bouar, and A Finel. Phase field methods and dislocations. Acta materialia, 51(1):17-30, 2003.

[30] Lucia Scardia and Caterina Ida Zeppieri. Line-Tension Model for Plasticity as the $\Gamma$-Limit of a Nonlinear Dislocation Energy. SIAM J. Math. Anal., 44(4):2372-2400, 2012.

[31] L. E. Shilkrot, R. E. Miller, and W. A. Curtin. Multiscale plasticity modeling: Coupled atomistics and discrete dislocation mechanics. J. Mech. Phys. Solids, 52:755-787, 2004.

[32] J. E. Sinclair. Improved atomistic model of a bcc dislocation core. J. Appl. Phys., 42:5231, 1971.

[33] J. E. Sinclair. Improved atomistic model of a BCC dislocation core. J. Appl. Phys., 42:5231, 1971.

[34] G. I. Taylor. The mechanism of plastic deformation of crystals. Part I. Theoretical. Proceedings of the Royal Society of London. Series A, Containing Papers of a Mathematical and Physical Character, 145(855), 1934.

[35] V. Vítek, R. C. Perrin, and D. K. Bowen. The core structure of $\frac{1}{2}(111)$ screw dislocations in BCC crystals. Philosophical Magazine, 21(173):1049-1073, 1970.

[36] Vito Volterra. Sur l'équilibre des corps élastiques multiplement connexes. Ann. Sci. École Norm. Sup. (3), 24, 1907.

[37] R.E. Voskoboinikov, S.J. Chapman, J.R. Ockendon, and D.J. Allwright. Continuum and discrete models of dislocation pile-ups. i. pile-up at a lock. Journal of the Mechanics and Physics of Solids, 55(9):2007 $2025,2007$.

T. Hudson, Mathematical Institute, University of Oxford, Oxford OX1 3LB, UK

E-mail address: thomas.hudson@maths.ox.ac.uk

C. Ortner, Mathematics Institute, Zeeman Building, University of Warwick, Coventry CV4 7AL, UK

E-mail address: c.ortner@warwick.ac.uk 